\title{
Behandlung der Wortbildungselemente in deutsch-tschechischen Übersetzungswörterbüchern
}

\author{
Martin Šemelík (Prag)
}

The aim of this paper is threefold. First, it aspires to discuss some general considerations regarding the treatment of word-forming elements in a bilingual dictionary. It is argued that not few lexicographic decisions are to be made rather independently of fluctuating linguistic postulates and that the user aspect and the function of the respective dictionary should be paid special attention to. Second, the paper examines the quantity and quality of the information provided on affixes in two German-Czech dictionaries. The analysis focuses on the entry components terminology, spelling, pronunciation, input and output units, sense distinction, translation equivalents, examples, cross-references, usage labels and productivity as well as on selectional criteria and information on affixes in the outside matter. The analysis reveals that the dictionaries under scrutiny show considerable weaknesses with respect to the information provided. Third, the paper briefly describes the treatment of the German prefix/circumfix Ge-...(-e) in the emerging Large German-Czech Academic Dictionary, an output of a project pursued at the Institute of Germanic Studies, Faculty of Philosophy \& Arts, Charles University in Prague. It is shown how contrastive analysis can be supplemented by a corpus-based view using new corpus-linguistic tools.

"Leicht hat es die Wortbildung nicht im Wörterbuch!" (Burgschmidt 2004: 183)

\section{Zur Einführung}

Dieser Text ist ein Output der Arbeiten am Projekt des Großen Akademischen Wörterbuchs Deutsch-Tschechisch (GAWDT), das unter Leitung von Marie Vachková am Institut für Germanische Studien der Philosophischen Fakultät der Karls-Universität in Prag entsteht (GAČR 405/09/1280, zur Projektbeschreibung cf. Vachková 2011). Auch wenn das GAWDT primär als ein elektronisches, wortartenspezifisch angelegtes Datenbanksystem konzipiert ist, fokussiert die das Projekt begleitende metalexikographische Forschung auch auf verfügbare gedruckte Wörterbücher des Sprachenpaars Deutsch-Tschechisch. Diese Auseinandersetzung mit Konzeptionen vergangener Jahrzehnte bzw. sogar auch Jahrhunderte ist ein Bestandteil metalexikographischer Reflexionen, aufgrund derer die Konzeption für die Behandlung der Wortbildung im GADWT erarbeitet wird.

Der vorliegende Beitrag setzt sich zum Ziel, (a) auf einige allgemeine Aspekte der lexikographischen Bearbeitung der Wortbildungselemente einzugehen (Kap. 1), (b) die 
Behandlung der Affixe in deutsch-tschechischen Wörterbüchern kritisch zu kommentieren (Kap. 2) und (c) die Behandlung der Affixe im GADWT am Beispiel des Präfixes/Zirkumfixes Ge-...(-e) zu skizzieren (Kap. 3).

\section{Allgemeine Aspekte der lexikographischen Bearbeitung der Wortbildungselemente}

\subsection{Wortbildungselemente in einem Übersetzungswörterbuch}

Daran, dass die Wortbildungselemente in Übersetzungswörterbücher Eingang finden sollen, wird gegenwärtig nicht mehr gezweifelt, zumal ein Übersetzungswörterbuch in der Regel auch die Rolle eines Lernerwörterbuchs übernehmen soll und somit didaktische Ziele zu verfolgen hat (cf. z. B. Šimečková 1987: 224, Vachková 2002: 121). Hinsichtlich der Behandlung der Wortbildungselemente sind in einem primär rezeptiven Übersetzungswörterbuch dabei folgende Aspekte zu berücksichtigen: (a) Dekodierung, (b) metalexikalischer Aspekt, (c) Übersetzung und eventuell auch (d) Sprachproduktion. Die Entscheidung, die Wortbildungszusammenhänge u. a.m. durch das Bewusstmachen von Wortbildungstypen und morphosemantischen Paradigmen wie Wortbildungsreihen und Wortbildungsnestern - wenn auch nur ausschnittsweise ${ }^{1}$ - darzustellen, entspricht der Erkenntnis, dass die Wörterbuchbenutzer durch Inventarisierung von Wortbildungselementen Einblicke in die Organisierung des jeweiligen Lexikonsegments bekommen können (metalexikalischer Aspekt), was besonders in denjenigen Wörterbüchern von Belang ist, in denen nicht nahe verwandte Sprachen kontrastiert werden. Den Benutzern werden dadurch Motivationsbeziehungen zwischen einzelnen Wortschatzeinheiten der Fremdsprache bewusst gemacht und sie bekommen Hilfe bei der Suche nach Äquivalenten einer nicht inventarisierten okkasionellen Wortbildung. Die Dekodierungswörterbücher können dabei auch bei fremdsprachiger Produktion, etwa als Kontrollinstanz, verwendet werden. Die Rolle der Wörterbuchartikel von Wortbildungselementen als Hilfsmittel für die Sprachproduktion ist jedoch zum Teil umstritten: "Für die Akzeptabilität [einer Neubildung - M. Š.] aber ist nicht allein die Modellgerechtheit entscheidend [...]. Über die Akzeptabilität in einem bestimmten Text, in einer bestimmten Situation entscheidet letztendlich der Hörer bzw. der Leser [...]. Die Risiken beim produktiven Umgang mit der Wortbildung sind also für den Lerner bedeutend größer als beim rezeptiven Umgang" (Poethe 1996: 190f.).

\subsection{Zum Verhältnis von Linguistik und bilingualer Lexikographie am Beispiel der sog. Affixoide}

Die Behauptung, dass die Lexikographie als "kulturelle Praxis, die die Erstellung von Wörterbüchern zum Ziel hat" (Engelberg/Lemnitzer 2009: 3) und Wortbildungsforschung viel gemeinsam haben, ist - fasst man die grundlegenden Fragen der (Meta)lexikographie wie z. B. Lemmaauswahl und Lemmaanordnung oder aus der umgekehrten Perspektive die Verwendung der Wörterbücher als Materialquellen für Wortbildungsforschung ins Auge - der Zustimmung sicher. "Nur sind bislang beide Seiten unzufrieden mit ihren Kooperationsergebnissen, dabei in besonderem Maße die praktische Lexikographie, denn sie

\footnotetext{
1 Die Diskrepanz zwischen Sprachkompetenz und Wörterbuch wird beispielsweise in Barz (2001: 86f.) thematisiert: "Insofern kann auch die Repräsentation der Wortschatzvernetzung in einem Wörterbuch, welchen Typs und Umfangs auch immer, nur ein bescheidener Ausschnitt aus muttersprachlichem Wissen sein".
} 
muss eine Vielzahl von Entscheidungen treffen, für die von der Wortbildungsforschung Lösungsangebote erwartet werden könnten, aber nicht vorliegen" (Barz 2000: 308).

Mit Fragen von grundlegender Bedeutung sind Lexikographen z. B. immer dann konfrontiert, wenn eine benutzerfreundliche Erfassung der sog. Affixoide angestrebt wird. Als Affixoide bzw. Halbaffixe oder Halbableiter werden in der Forschungsliteratur die Wortbildungselemente bezeichnet, die sich in Folge ihrer ausgeprägten Reihenbildung als Erst- oder Zweitglieder eines Kompositums in der Entwicklung zu einem semantisch unselbständigen, affixartigen Element zu befinden scheinen (cf. Bußmann 2008: 12, inter alia) - z. B. -freundlich in eine hautfreundliche Seife, Affen- in Affentempo usw. Der Affixoidbegriff ist dabei in der heutigen Diskussion immer noch weitgehend umstritten. In Fleischer/Barz (2007), Donalies (2005) oder Fandrych (2011) wird er als überflüssig empfunden, anders dagegen aber in Ascoop (2005), Elsen (2009) oder Müller (1989). Die Kritik am Affixoidbegriff kann in Anlehnung an Schmidt (1987: 81) wie folgt zusammengefasst werden: Die Bedeutungsabweichung der sog. Affixoide sei eine ganz normale Erscheinung jeder Zeichenverwendung. Das gleiche Sprachzeichen könne in unterschiedlichen sprachlichen Umgebungen auch unterschiedliche aktuelle Bedeutungen, d. h. unterschiedliche Aktualisierungen von Teilen seiner Bedeutung aufweisen. Unterschiede zwischen dem syntaktischen Verhalten der Wörter im Satz und ihrem Auftreten in komplexen Wörtern dürften dabei nicht zum Kriterium für einen wechselnden Morphemstatus erhoben werden, denn es gehöre gerade zu den spezifischen Leistungen der Wortbildung, dass sie ganze Sätze in einer Kombination zusammenfasse.

Dass die reihenbildenden Erst- und Zweitglieder aber doch über "besondere Eigenschaften" (Poethe 1996: 195) verfügen, wird mittlerweile auch von denjenigen eingeräumt, die auf den Affixoidbegriff ansonsten verzichten: "Die Kategorie Affixoid ist in der Wortbildungsforschung umstritten, und zwar weniger wegen des Charakters der fraglichen Einheiten als Übergangsstadium zwischen Wort und Affix, als vielmehr hinsichtlich der Notwendigkeit, dieses Zwischenstadium kategoriell und damit terminologisch gesondert zu fassen [...]. Grundsätzlich scheint gegenwärtig die strikte Ablehnung der Kategorie Affixoid einer differenzierten Sicht Platz zu machen, indem bestimmte Kompositionsglieder oder Derivationsteile aufgrund spezifischer suprasegmentaler, morphologischer und semantischer Eigenschaften doch den Zwischenstatus zugesprochen bekommen [...]" (Barz 2002: 112).

Die bisher vernachlässigte lexikographische Bearbeitung dieser Wortbildungselemente z. B. in Übersetzungswörterbüchern des Sprachenpaars Deutsch-Tschechisch steht im Kontrast zu dem regen Interesse, dessen sich diese Erscheinungen in einsprachigen Wörterbüchern des Deutschen erfreuen. Die Kompositakonstituenten des Typs -freundlich, -frei, -reich u. Ä. sind, von ihrer terminologischen Erfassung abgesehen, in ein deutsch-tschechisches Wörterbuch als selbständige Wörterbuchartikel aufzunehmen, weil sie einen erheblichen Teil der schweren, d. h. der schwer übersetzbaren Wörter abdecken (cf. Vachková 2006: 169). Dabei stellt sich ähnlich wie bei den Affixen u. a. die Frage, wie und an welcher Stelle diese Kompositakonstituenten im Wörterbuch dargestellt werden sollen. In Prćić (2008: 16f.) wird diese Frage wie folgt beantwortet: "[Not] belonging to the categories of either synchronic suffixes or synchronic FCFs [Final Combining Forms - M. Š.] are adjectives like -free, -rich [...] -friendly [...], which occur in adjectival compositions like sugar-free, feature-rich [...] user-friendly [...]. [T]here is no reason for according these elements separate entries in 
dictionaries, because they do not constitute separate elements; instead these elements should be included in the entries of their source words, where they morphologically and semantically indubitably belong". Auf Einzelheiten der praktischen Umsetzung dieser Konzeption anhand eines konkreten Wörterbuchartikels wird hierbei aber leider nicht eingegangen.

Diese Problematik hat dabei eine allgemeinere Dimension, die hier jedoch nur gestreift werden kann. Das "Aufeinanderangewiesensein" (Barz 2000: 308) von Wortbildung und Lexikographie ist m. E. nicht so zu verstehen, dass das Wörterbuch eine Art Projektionsfläche darstellen sollte, auf der sich die Postulate der theoretischen Linguistik immer ganz getreu widerzuspiegeln hätten. Linguistische Konzeptionen sind keineswegs mechanisch auf die praktische Lexikographie übertragbar, sondern sie sollten in die lexikographische Theorie und Praxis integriert werden, und das in einer adäquat eingerichteten Gestalt (dazu allgemein neuerdings Tarp 2009). Die Ansicht, dass "[e]s nicht zu erwarten [ist], dass Wörterbuchverfasser die jeweils diskutierten Theorien für Semantik und/oder Wortbildung voll zur Kenntnis nehmen oder gar voll adäquat bei Zehntausenden von Wörtern einarbeiten könnten" (Burgschmidt 2004: 181), trifft auch für ein universitär verankertes Projekt wie das GAWDT zu. Lexikographische Entscheidungen müssen einerseits Resultate der linguistischen Forschung berücksichtigen, andererseits aber auch den Benutzeraspekt und die Funktion des Wörterbuchs in Betracht ziehen, sodass der Benutzer mit seinen Anforderungen im Zentrum steht. Starr auf zu einem bestimmten Zeitpunkt gerade aktuellen Postulaten und Ergebnissen der theoretischen Wortbildungsforschung zu beharren, würde z. B. im Falle der lexikographischen Bearbeitung der reihenbildenden Zweitglieder bedeuten, dem Benutzeraspekt weniger Bedeutung einzuräumen, als ihm zukommt. Gerade im Falle einer stark praktisch orientierten Disziplin wie Lexikographie sind es viel mehr praktische bzw. didaktische Gründe - etwa das Fehlen der reihenbildenden Erst- und Zweitglieder in einer der lexikographisch zu erfassenden Sprachen - als systematisch-kategorielle Gesichtspunkte, die die "Wiederbelebung" bzw. Einführung des Affixoid-Begriffs in einigen bilingualen Wörterbüchern begünstigen dürften. Aus dieser Perspektive erscheint mir H. Elsens "Nur was benannt wird, kann auch verstanden werden" (cf. Elsen 2009) nicht unberechtigt. Auch hier zeigt sich, dass die Lexikographie weder Teil der angewandten Linguistik noch der Lexikologie ist, sondern eine eigenständige Disziplin, die eigene Theorien entwickelt (cf. Bergenholtz 2000: 19) und Entscheidungen treffen muss, die mit strikt linguistischen Fragestellungen oft wenig gemeinsam haben, z. B. Layoutfragen u. Ä. (cf. ebd.). Die Unzufriedenheit mit den Kooperationsergebnissen der Wortbildungsforschung und der Lexikographie seitens der Lexikographen ist dabei m. E. zum Teil eben auch dadurch gegeben, dass diese Tatsache nicht voll zur Kenntnis genommen wird. ${ }^{2}$

\subsection{Lexikographische Behandlung der Wortbildungselemente}

Mit Irma Hyvärinen (2000: 39) kann hinsichtlich der Auswahl der ins Wörterbuch aufzunehmenden Wortbildungselemente festgehalten werden, dass deren Wahl "kaum nach

\footnotetext{
${ }^{2}$ Die Schwierigkeit scheint auch darin zu bestehen, dass z. B. die Ergebnisse der kontrastiven Wortbildung lexikographisch nicht ohne Weiteres umsetzbar sind, zumal sie entweder systemorientiert und stark verallgemeinernd sind oder zu speziell bearbeitet in Form von Fallstudien vorliegen. So sucht der nichtdeutsche Lexikograph nach den Vorbildern im Bereich der einsprachigen Lexikographie des Deutschen. Die Umsetzung der angeregten Bearbeitung setzt jedoch auch umfangreiche Analysen der tschechischen Wortbildung voraus, die zuerst sehr detaillierten Materialanalysen zu entnehmen sind.
} 
einem einzigen Prinzip erfolgen kann und dass viele Kompromisse nötig sind, wobei die Systematik im Hinblick auf den Wortbildungsstatus eines Elementes nicht unbedingt immer das relevanteste Aufnahmekriterium ist".

Kontroversen über die lexikographische Behandlung der Wortbildungselemente betreffen v. a. die Fragen, in welchem Umfang diese Einheiten ins Wörterbuch aufgenommen werden sollen, an welcher Stelle sie zu behandeln sind (im Hauptregister oder in einem separaten Anhang im Vor- oder Nachspann) bzw. wie die oft eher unsystematischen Äquivalenzverhältnisse gehandhabt werden sollen usw. (cf. Hyvärinen 2000: 33f.).

Den Autoren eines Printwörterbuchs sind angesichts des allgemeinen Platzmangels zahlreiche Einschränkungen auferlegt, was u. a. zur Folge hat, dass eine maximale Aufnahme von Wortbildungselementen in der Regel ausbleiben muss. Praktische Problemlösungen bei der Auswahl der aufzunehmenden Wortbildungselemente werden in Hyvärinen (2000) angeboten. Die Aufnahme eines Wortbildungselementes begünstigen v. a. dessen hohe Produktivität, Besonderheiten in kontrastiver Sicht (z. B. das Fehlen von Verbalpräfixen im Finnischen im Vergleich zum Deutschen), relativ leichte Segmentierbarkeit sowie Auffindbarkeit (Präelemente sind leichter segmentierbar als Endelemente; unter den Endelementen sind solche leichter auffindbar, deren morphologische Grenze mit der Silbengrenze übereinstimmt) (cf. Hyvärinen 2000: 40-46). "Von den Präelementen sollen am ehesten die kurzen extra lemmatisiert werden, denn die betreffenden Wortbildungsprodukte 'verlieren' sich unter der alphabetischen Reihenfolge" (Hyvärinen 2000: 41). Dass mit einer solchen Vorgehensweise, die u. a. eine systematische Erfassung der Äquivalenzverhälnisse bei allen zu beurteilenden Wortbildungselementen voraussetzt, notwendigerweise ein enormer Zeitaufwand verbunden ist, liegt auf der Hand, doch "[d]er gewählte Weg von einer maximalen Bestandsaufnahme zu einer knappen Liste [...] entspricht [...] dem wissenschaftlichen Wissen überhaupt: Erst ein Gesamtbild lässt das Wesentliche herausragen" (Hyvärinen 2000: 50).

Eine andere Lösung wird in Brdar-Szabó (1996) angeboten, wo für die "Verbannung" (BrdarSzabó 1996: 88) der Wortbildungselemente aus dem Hauptregister in die Umtexte plädiert wird, und zwar mit folgenden Argumenten (cf. Brdar-Szabó 1996: 85-87):

(a) Die anderssprachigen Wörterbuchbenutzer seien nicht in der Lage, die fremden Wortbildungsprodukte sinnvoll $\mathrm{zu}$ segmentieren und entsprechend an der richtigen Stelle nachzuschlagen.

(b) Durch die Lemmatisierung der Wortbildungselemente werde deren Lexemstatus suggeriert.

(c) Die Darstellung der Bedeutungsnischen bei polysemen Wortbildungselementen erwecke den Anschein, dass die Wortbildungselemente als gebundene Wortbildungsmorpheme von vornherein statisch fixierte Bedeutungen hätten. Der Bedeutungsanteil der Wortbildungselemente sei aber erst im Nachhinein aufgrund der Kenntnis der Gesamtbedeutung des Wortbildungsproduktes zu erschließen.

(d) Durch Lemmatisierung der Suffixe werde das alphabetische Prinzip verletzt.

(e) Äquivalenzverhältnisse können sehr unsystematisch sein. 
Auf einzelne Punkte ist hier kurz einzugehen.

Ad (a):

In diesem Punkt scheint m. E. eine differenziertere Perspektive erforderlich zu sein. Bei professionellen Übersetzern, die im entsprechenden Wörterbuchartikel nach Übersetzungsmöglichkeiten der ins Wörterbuch nicht aufgenommenen, doch beispielsweise in Zeitungstexten durchaus häufig auftretenden Okkasionalismen suchen ${ }^{3}$, ist sicher mit einer genügend entwickelten Wortbildungskompetenz zu rechnen. Kann die Fähigkeit, lexikalische Einheiten der Ausgangssprache sinnvoll zu segmentieren, bei den Wörterbuchbenutzern jedoch nicht vorausgesetzt werden, dann macht aber die Verlegung von Wortbildungselementen in Umtexte sowieso wenig Sinn, denn es leuchtet nicht ein, warum die Wörterbuchbenutzer dann die Wortbildungselemente eigentlich irgendwo suchen sollten, darüber hinaus in Umtexten, die von den meisten Wörterbuchbenutzern nur selten zur Kenntnis genommen werden (cf. Engelberg/Lemnitzer 2009: 88). Auch in diesem Fall lohnt es sich aber, die Wortbildungselemente in das Wörterbuch aufzunehmen. Schlagen die Wörterbuchbenutzer unter trinkbar nach, können sie mittels eines Verweises zum Wörterbucheintrag -bar weitergeleitet werden, der ihnen Angaben zur strukturellen Beschaffenheit der Wortbildungsprodukte auf -bar anbietet. Dadurch kann die Wortbildungskompetenz der Wörterbuchbenutzer gesteigert werden, sodass sie die morphologisch komplexeren Wortbildungsprodukte bei künftiger Sprachrezeption leichter segmentieren können. ${ }^{4}$ Ob entsprechende Wörterbuchartikel dann im Hauptregister oder in Umtexten untergebracht sind, ist m. E. eine Frage von untergeordneter Bedeutung.

Ad (b):

Den Wortbildungselementen unterhalb der Wortebene wird tatsächlich keine selbständige lexikalische Bedeutung zugeschrieben, weshalb ihr Lexemstatus aus wortbildungstheoretischer Sicht auch anfechtbar ist. Es trifft aber andererseits zu, dass Wörterbücher im Hauptregister als Lemmata oft auch solche Zeichen wie z. B. " $\Omega$ " u. Ä. führen (siehe z. B. das DUW-P), deren Lexemstatus ebenfalls nicht unbestritten ist. Außerdem sei hier an Fälle erinnert wie die Lyrik - der Lyriker, naiv - der Naivling usw., in

\footnotetext{
${ }^{3}$ Bis ein Drittel der in einem Zeitungstext vorkommenden Wortbildungen sind in Wörterbüchern nicht inventarisiert (cf. Wellmann 1995: 400).

${ }^{4}$ Der Benutzeraspekt ist hierbei von zentraler Bedeutung. Nicht alle Benutzer haben die gleichen Bedürfnisse und die gleiche Fähigkeit, das sprachliche Material zu analysieren, was auch auf das GAWDT zutrifft (cf. Šemelík 2012: 138). Die Frage, ob die Benutzer der zweisprachigen Wörterbücher auch das suchen, was in einsprachigen Wörterbüchern bzw. Grammatiken oder einschlägigen Monographien zur Wortbildung steht, sei an dieser Stelle als Problemstellung formuliert. Nach Bergenholtz (2001) ist die schon vor längerer Zeit aufgestellte Forderung nach Benutzeruntersuchungen eine Forderung, "die fortbesteht, die aber nur in geringem $\mathrm{Ma} ß$ von den größtenteils theoriearmen blinden Einsammlern von Fragebögen zu wirklich wesentlichen neuen Einsichten oder überhaupt zu glaubhaften Bestätigungen vorliegender Vermutungen geführt hat. Etwas relevanter erscheinen die Wörterbuchbenutzungsprotokolle [...], die Wörterbuchbenutzer direkt nach konkreten Nachschlagehandlungen aufgezeichnet haben. Aber auch diese Protokolle sind mit einigen Problemen behaftet [...]. Weit mehr Einsicht in die Bedürfnisse der Wörterbuchbenutzer sind [...] nicht aus den empirischen, sondern aus den vorwiegend theoretischen Überlegungen abzuleiten" (Bergenholtz 2001: 15). So geht z. B. die GAWDTRedaktion davon aus, dass Übersetzungswörterbücher nicht nur im Falle eines Textproblems konsultiert, sondern auch zwecks Wissensbeschaffung herangezogen werden (können), wobei die Information selbst das genuine Ziel der Nachschlagehandlung sein kann (kognitive Funktion, cf. die im Rahmen der Aarhuser lexikographischen Schule entwickelte Funktionslehre v. a. in Tarp 1995). Aus dieser Perspektive erscheint z. B. die Berücksichtigung der Monographien zur Wortbildung im Falle der lexikographischen Beschreibung der Wortbildungselemente im GAWDT plausibel.
} 
denen die jeweiligen Affixe Veränderungen der kategoriellen Bedeutung bewirken und folglich wohl doch nicht für völlig frei von jeder Bedeutung gehalten werden können (ausführlicher cf. z. B. Donalies 2005: 37).

Ad (c):

Ähnliches gilt aber auch für Lexeme. Die Vorstellung, dass den Lexemen einfach statisch eine fixierte Bedeutung "anhängt", ist aufzugeben. Auch ihre Bedeutung kann erst im Nachhinein durch Bezugnahme auf eine höhere Ebene, etwa die syntagmatische oder sogar textuelle, eruiert werden. Die durchnummerierten Lesarten in entsprechenden Wörterbuchartikeln erwecken darüber hinaus den Anschein, dass die Lexeme eine objektiv gegebene Anzahl von Lesarten hätten. Dass dies nicht der Wirklichkeit entspricht, bezeugt u. a. die Tatsache, dass ein bestimmtes Bedeutungsspektrum in verschiedenen, doch in Bezug auf deren Umfang und Zweck vergleichbaren Wörterbüchern auf unterschiedliche Art und Weise segmentiert wird. Nun sind dies keine Argumente dafür, diese Lexeme lexikographisch an anderer Stelle als im Hauptregister darzustellen.

$\operatorname{Ad}(\mathrm{d})$ :

Die Suffixe -heit oder -lich findet man z. B. im DUW-P zwischen Heister und heiter, resp. zwischen libysch und Licht. Wie dies mit dem alphabetischen Prinzip kollidieren sollte, ist nicht klar. Die Wortbildungsprodukte, die in diesen Wörterbuchartikeln angeführt sind, haben m. E. denselben Status wie Beispielsyntagmen und -sätze in Lexemstichwörtern. Es sind keine Lemmata, sodass sie der makrostrukturellen Anordnung nicht unterliegen und das alphabetische Prinzip nicht verletzen. Nicht einmal Allomorphe erweisen sich in dieser Hinsicht als problematisch. Alle Allomorphe können in einem Haupteintrag angeführt werden. An der Stelle, an der sie sonst dem Alphabet nach behandelt werden sollten, kann ein Verweislemma stehen, das den Benutzer zum Haupteintrag weiterleitet (z. B. -ativ verweist auf -iv, wo -ativ mit behandelt wird).

$\operatorname{Ad}(\mathrm{e})$ :

Die oft fehlende Systemhaftigkeit der Äquivalente unterhalb der Wortebene bedeutet nicht, dass auf die Inventarisierung von Wortbildungselementen im Hauptregister notwendigerweise verzichtet werden muss. Der Behauptung, dass die Übersetzung der ausgangssprachlichen Angaben und Bedeutungserklärungen unter Beibehaltung der deutschen Polysemiestrukturierung als Notlösung mit dem Konzept eines zweisprachigen Wörterbuches nicht zu vereinbaren sei (cf. Brdar-Szabó 1996: 86), kann m. E. nicht ohne Vorbehalt zugestimmt werden, denn das Fehlen von direkten Äquivalenten kann auch auf der Lexemebene beobachtet werden. Man denke beispielsweise an Fälle wie Percht oder Krampus in der deutsch-englischen Relation, Oktoberfest und Eintopf in der deutschtschechischen u. Ä. Trotzdem gibt es gute Gründe dafür, diese Wortschatzeinheiten in das Wörterbuch aufzunehmen. Mit der Verlagerung der Wortbildungselemente in die Umtexte ist man des Problems der fehlenden Systemhaftigkeit der Äquivalente ja auch nicht enthoben. Ähnliche Maßnahmen sind deshalb m. E. durchaus möglich, obwohl nicht unbedingt nötig, denn erhebliche Vorteile für die Wörterbuchbenutzer können damit kaum erzielt und die meisten Probleme mit der lexikographischen Erfassung der Wortbildungselemente nicht gelöst werden. Vorteilhaft ist ihre Verlagerung vielleicht nur insofern, als die Benutzer die 
Wörterbuchartikel einer bestimmten Klasse von Wortbildungselementen im Wörterbuch an einer einzigen Stelle finden, sodass sie sich über sie problemlos Überblick verschaffen können, ohne mühsam im Wörterverzeichnis blättern zu müssen. Um dieser Forderung gerecht $\mathrm{zu}$ werden, kommt auch eine doppelte Inventarisierung in Frage, wie sie z. B. im OALD praktiziert wird. Dass dies aber mit dem Ökonomieprinzip eines Printwörterbuchs unvermeidlich kollidiert, liegt auf der Hand.

\subsection{Wortbildungselemente in einem Übersetzungswörterbuch - ein Angabenkatalog}

\subsubsection{Umtexte}

In Umtexten sind die Termini Wortbildungselemente, Affix(oid)e usw. anhand geeigneter Beispiele zu erklären. Es ist auch auf lexikographische Entscheidungen bei ihrer Inventarisierung (Umgang mit Allomorphen, Gestaltung der grammatischen, stilistischen und semantischen Daten, Repräsentanz der Beispiele etc. - cf. Barz 2003: 384) einzugehen. Eine Liste der inventarisierten Wortbildungselemente kann den Begriffsumfang des Terminus Wortbildungselement verdeutlichen, einen Überblick über die Wortbildungsmöglichkeiten der entsprechenden Sprache bieten, Assistenz bei der Worterschließung geben u. a. m. (cf. ebd.).

\subsubsection{Artikelgestaltung}

Im Falle eines Übersetzungswörterbuchs sind in Wörterbucheinträgen der Wortbildungselemente folgende Angaben unterzubringen 5 :

(a) Terminologie: Das Wortbildungselement ist der entsprechenden Kategorie der Wortbildungselemente zuzuordnen. Obwohl es sicher zutrifft, dass die meisten Wörterbuchbenutzer diese Angaben kaum einzuschätzen vermögen (cf. Barz 2003: 387 in Anlehnung an Mugdan 1984), können sie für philologisch interessierte Benutzer nützlich sein. ${ }^{6}$

(b) Formseite, Wortbildungsbasis, Wortbildungsprodukt und grammatische Angaben: Varianten des Wortbildungselementes (vgl. z. B. Ge-...(-e) in Gebell und ...-ge-...-e in Herumgehopse) sind zu berücksichtigen sowie Angaben zur Wortartzugehörigkeit sowohl der Wortbildungsbasis als auch des Wortbildungsproduktes einschließlich der Angaben zu morphosemantischen Eigenschaften der Basis, die v. a. dann von Bedeutung sind, wenn das Wörterbuch seinen Benutzern auch bei der Sprachproduktion behilflich sein will. In die Wörterbuchartikel sind grammatische Angaben aufzunehmen (z. B. bei Substantiven die Formen des Gen. Sg. und Nom. Pl.).

\footnotetext{
5 Zum Teil in Anlehnung an Prćić (1999), wo ein Angabenkatalog für Affixartikel in englischen Lernerwörterbüchern erarbeitet worden ist.

${ }^{6}$ Eben dieser Aspekt betrifft das sich auch an die Akademiker wendende GAWDT. Nicht nur im Falle der sog. Affixoide ist dabei mit terminologischen Schwierigkeiten zu rechnen. Definiert man die Präfixe als immobil (cf. z. B. Donalies 2005: 26), dann stellt sich die Frage, wie die Elemente wie $a b$-, vor- usw. etwa in absehen oder vorstellen u. Ä. terminologisch fixiert werden sollten. Eisenberg (1998: 254) verwendet den Begriff "Partikelverb", Thurmair (1996: 166) setzt hierbei eine eigene Wortbildungsform an, die sie "Konstitution" nennt, während z. B. Šimečková (1996: 148) den Begriff "Präverbfügung" verwendet (zu einer Übersicht der gängigen Terminologie cf. Donalies 2005: 28-30 und Šimečková 1994: 29-33). Im Falle des GAWDT würde ich für die Termini "trennbares" und "untrennbares Präfix" plädieren, unter denen diese Erscheinungen in tschechischen Lehrbüchern und Grammatiken des Deutschen gewöhnlich firmieren und an die die tschechischen
} 
(c) Aussprache: Im Wörterbuchartikel sollten Angaben zum Wortakzent, ggf. auch phonologische Transkription untergebracht werden, falls dies aus kontrastiver Perspektive sinnvoll ist. In einem deutsch-tschechischen Wörterbuch für tschechische Muttersprachler würde die Transkription z. B. bei er-, ver-, zer- oder bei -lich in Frage kommen, weil es im Tschechischen weder das vokalisierte $r$ noch den sog. ich-Laut gibt, und tschechische Muttersprachler mit der Aussprache entsprechender Wortbildungsprodukte folglich Schwierigkeiten haben. Dasselbe betrifft auch den Transfer bei dem Konflikt der progressiven und regressiven Assimilation (vgl. die tschechische Aussprache von -ismus als [-Izmos]) sowie bei der Auslautverhärtung (vgl. die Tendenz der tschechischen Muttersprachler z. B. $a b$ - in abnehmen als ['ab-] anstatt von ['ap-] auszusprechen; en détail cf. Schmidt/Vachková 2009).

(d) Wortbildungsbedeutung, Äquivalenz und Beispiele: Die Erklärung der Wortbildungsbedeutung kann durch knappe Kommentare erfolgen. Weisen Äquivalenzverhältnisse zumindest zum Teil systemhafte Züge auf, sollten Äquivalente angeführt werden. Als Beispiele sind bei produktiven Wortbildungsmodellen morphosemantisch noch voll motivierte Wortbildungsprodukte zu bevorzugen.

(e) Paradigmatische Relationen: Es ist auf synonymische und antonymische Wortbildungselemente $\mathrm{zu}$ verweisen. Detaillierter kann auf die Problematik der konkurrierenden Wortbildungselemente in der Wörterbuchgrammatik eingegangen werden.

(f) Diasystematische Angaben: Es handelt sich v. a. um Stilschicht- (gehoben, informell usw.) und Konnotationsangaben (abwertend u. Ä.).

(g) Produktivität: Die Produktivitätsangaben ermöglichen Aufschlüsse über die Fähigkeit von Wortbildungsmodellen bzw. Wortbildungselementen zur Neubildung sprachlicher Ausdrücke. Die Wortbildungsproduktivität stellt dabei eine gradiente Erscheinung dar, der im Wörterbuch Rechnung zu tragen ist, indem sie mithilfe einer Dreistufenskala (etwa sehr produktiv/begrenzt produktiv/nicht produktiv - cf. z. B. Poethe 1996: 203f., Prćić 1999: 268) markiert wird. ${ }^{7}$

Deutschlerner folglich gewöhnt sind. Auf die Probleme, die mit der jeweiligen Terminologie verbunden sind, könnte in der Wörterbuchgrammatik eingegangen werden.

7 Die Beurteilung der Produktivität eines Wortbildungselementes bzw. dessen Modelle sollte in einem korpusgesteuerten Wörterbuch von einem Zusammenspiel qualitativer (Erfassung der Restriktionen möglicher Wortbildungsbasen eines bestimmten Wortbildungsmodells, siehe unter (b) oben) und quantitativer (Erfassung des Produktivitätsgrades eines Modells) Korpusanalyse ausgehen. Die oft nur intuitiven Urteile über die Produktivität eines Wortbildungsmodells sind durch statistisch untermauerte Angaben zu ersetzen. Entsprechende Verfahren werden in Lüdeling/Evert (2003) am Beispiel des Suffixes -lich veranschaulicht. Nützliche Informationen zur Produktivität einzelner Wortbildungselemente können auch dem Archiv der Wortwarte, einer Sammlung von Neologismen (Projektleitung: Lothar Lemnitzer, Berlin-Brandenburgische Akademie der Wissenschaften) entnommen werden (vgl. unter www.wortwarte.de/index.html). Im GAWDT wird ein Wortbildungselement in der Regel dann als produktiv erklärt, wenn ein Vergleich zwischen dem Inventar der bestehenden Wörterbücher und den Neologismen in Korpora (v. a. DeReKo - vgl. Kap. 3.2) diesen Schluss zulässt oder wenn das jeweilige Wortbildungselement in den verwendeten Sekundärquellen (vgl. ebd.) als produktiv markiert ist. Dass die lexikographische Erfassung der Produktivität eine äußerst schwierige Aufgabe ist, die in einem selbständigen Aufsatz behandelt werden sollte, muss nicht betont werden. 


\section{Affixe in vorliegenden deutsch-tschechischen Wörterbüchern}

Von insgesamt siebzehn deutsch-tschechischen bilingualen (bzw. bilingualisierten) Wörterbüchern von (mittel)großem Umfang ${ }^{8}$, die seit $1802^{9}$ herausgegeben worden sind, sind es nur Encyklopedický německo-český slovník (Enzyklopädisches Deutsch-böhmisches Wörterbuch, 1913-1935, weiter nur STERZ) von J. V. Sterzinger und Duden - Německý výkladový slovník s českými ekvivalenty (Duden - Deutsches Wörterbuch mit tschechischen Äquivalenten, erschienen 1993, eine bilingualisierte Version vom D-BWB, weiter nur DUDEN) von W. Müller et al., die ihren Benutzern Angaben zu Wortbildungselementen in selbständigen Wörterbuchartikeln anbieten. Für eine exemplarische Betrachtung sind hier Wörterbuchartikel der Affixe Ge-...(-e), -sam und ent- herausgegriffen worden (siehe Anhang I). Dank der facettenreichen Beschaffenheit dieser Affixe ${ }^{10}$ können ihre Wörterbuchartikel in Bezug auf das in Kap. 2 ausgearbeitete Angabeninventar als relativ repräsentativ gelten und somit eine tiefere Einsicht in die Behandlung der Affixe in beiden Wörterbüchern ermöglichen. Falls es als erforderlich erscheint, wird in Anmerkungen auf Besonderheiten auch anderer Wörterbuchartikel aufmerksam gemacht. Die hier vorgenommene quantitativqualitative Analyse erfolgt dabei in zwei Schritten (in Anlehnung an Prćić 1999: 207): 1. Es wird ein vorauszusetzendes (in mancherlei Hinsicht sicher noch ergänzbares) Angabenangebot formuliert, welches die Wörterbuchartikel enthalten sollten. 2. Dieses Angebot wird dann mit der Darstellung entsprechender Phänomene in beiden Wörterbüchern verglichen. Die Umtexte beider Wörterbücher werden auf die Definition des Begriffs "Affix" sowie auf die Informationen zu Entscheidungen bei der Affixinventarisierung hin untersucht.

\subsection{Umtexte und Affixselektion}

Die Umtexte des STERZ enthalten weder eine Definition des Begriffs Affix noch die Information, dass Wortbildungselemente in das Wörterbuch aufgenommen worden sind. Als Kontrollmenge für das Zusammenstellen einer Liste der inventarisierten Affixe dient hier das in Fleischer/Barz (2007: 36f.) vorliegende Affixinventar. Von den dort als Affixe bezeichneten Wortbildungselementen enthält das STERZ die folgenden:

(a) Präfixe: ab-, an-, auf-, aus-, be-, bei-/Bei-, dar-, ent-, er-, ge-/Ge-, hyper-, los-, miss-, nach-/Nach-, ob-/Ob-, sub-/Sub-, super-/Super-, über-, um-, un-, unter-/Unter-, ur-,Ur-, ver/Ver-, vor-/Vor-, zer-, zu-/Zu-

(b) Suffixe: -bold, -haft, -halben, -halber, -heit, -keit, -lei, -lein, -lich, -ling, -lings, -los, -schaft, -ung, -wärts, -weg, -weise

\footnotetext{
${ }^{8}$ In Anlehnung an Čermák (1995: 233) gilt hier Folgendes: Wörterbücher von mittlerem Umfang umfassen ca. 35 000-50 000 Wörterbuchartikel, Wörterbuch von großem Umfang dann in der Regel mehr als 80000 Wörterbuchartikel.

${ }^{9}$ Der Anfang des betreffenden Zeitraums wird mit dem Jahr 1802 gesetzt, dem Erscheinungsjahr des ersten Bandes des Deutsch-böhmischen Wörterbuchs von Dobrovský. Entscheidend hierfür ist ein soziolinguistisches Kriterium. Erst Dobrovský gelang es, nachdem die tschechische Sprache im Zuge der Germanisierungsprozesse im 17. und 18. Jh. um 1750 ihren Tiefpunkt erreicht hatte (cf. Skála 1994: 9), eine neue Norm der tschechischen Schriftsprache zu begründen, sodass das Tschechische in Bezug auf seine gesellschaftlich-kommunikative Leistungsfähigkeit dem Deutschen erneut gleichgestellt werden konnte.

10 Es handelt sich um Affixe, deren einzelne Wortbildungsreihen sich durch diastratische und diaevaluative Markiertheit kennzeichnen. Dabei berühren sie sich synonymisch mit anderen Wortbildungselementen, zu anderen werden sie dagegen antonymisch verwendet.
} 
Schon bei einem oberflächlichen Blick fällt z. B. das Fehlen von -bar, -chen, -ei/-erei, -er, -igkeit ${ }^{11}$, -tum u. a.m. auf, um nur einige von den nicht aufgenommenen Wortbildungselementen zu nennen.

Der Wortbildungsproblematik ist im DUDEN ein beträchtlicher Teil des Vorworts ("Předmluva", S. 3-5) gewidmet. Im Vordergrund stehen dabei aber v. a. die Affixoide. Zu Affixen erfahren die Wörterbuchbenutzer nur, dass es Wortbildungselemente gibt, die so genannt werden, wie etwa herbei- und -bar (cf. S. 3), es werden aber keine weiteren Beispiele angeführt. Eine Definition der Begriffe "Präfix" und "Suffix" erfolgt im Glossar der verwendeten linguistischen Termini ("Přehled použitých jazykovědných termínů", S. 805) im Nachspann des Wörterbuchs. In den Benutzungshinweisen ("Práce s heslem", S. 7) wird auf die Affixartikel nicht aufmerksam gemacht. Die Lemmaauswahl im DUDEN stimmt mit dessen Vorlage D-BWB überein. In die Umtexte ist eine Liste aller lemmatisierten Wortbildungselemente eingefügt worden. Stellt man die im DUDEN als Präfixe oder Suffixe bezeichneten Wortbildungselemente der Liste in Fleischer/Barz (2007, 36f.) gegenüber, ergeben sich $u$. A. folgende Unterschiede (Auswahl):

(a) bio-, semi-/Semi-, pseudo-/Pseudo-, Mini-, neo-/Neo- gelten in Fleischer/Barz als Konfixe, im DUDEN als Präfixe.

(b) -werk ist in Fleischer/Barz als Suffix ausgewiesen, im DUDEN als Suffixoid.

(c) folgende Wortbildungselemente sind nur im DUDEN (als Affixe) aufgelistet: -inski, pan-/Pan-, syn-/Syn-.

\subsection{Artikelgestaltung}

\subsubsection{Terminologie ${ }^{12}$}

\section{Zu erwarten:}

(a) Ge-: Präfix, Varianten Ge-...-e/-ge-...-e: Zirkumfix (b) -sam: Suffix (c) ent-: Präfix bzw. untrennbares Präfix o. Ä.

\section{Istzustand:}

(a) in beiden Wörterbüchern nur als Präfix bezeichnet, (b) in beiden Wörterbüchern wie oben dargestellt, (c) in beiden Wörterbüchern als Präfix bezeichnet; die Angabe zur Untrennbarkeit nur im STERZ13

\subsubsection{Formseite, Wortbildungsbasis und -produkt, grammatische Angaben}

\section{Zu erwarten:}

(a) bei allen Affixen entsprechende Graphemsequenz

\footnotetext{
11 -igkeit ist weder selbständig inventarisiert noch als Variante bei -keit angeführt.

12 Im STERZ weist die Zuordnung zu einzelnen Kategorien verschiedene Abweichungen auf. Bei unter-/Unter-, -heit, -los, sub-/Sub-, super-/Super-, -wärts, -weg wird keine Kategorisierung vorgenommen. Miss-/miss- ist als Bestimmungswort bezeichnet. Als Kompositionsglieder sind -bold und -lei (Zahlwort), als Partikeln ("částice") halben und -halber aufgefasst.

${ }^{13}$ Bei er- fehlt jedoch die Angabe zur Untrennbarkeit.
} 
(b) bei Ge- Varianten Ge-...-e und ...-ge...-e sowie die Angabe, dass das Suffix $-e$ nach unbetontem -e-der Basis oft fehlt (wimmeln-Gewimmel)

(c) Ge-...(-e): Basis: Verb (Restriktionen: in der Regel nicht reflexive Verben, Modalverben und Verben mit dem Suffix -ieren) oder Subst. (mit Bedeutungsdifferenzierung - Kollektiva); Produkt: Subst. (Neutrum)

(d) Ge-...(-e): grammatische Angaben: -s, 0 o. Ä. für Gen. Sg. und Nom. Pl.

(e) -sam: Basis: Verb und Subst., Produkt: Adj.

(f) ent-: Basis: Verb, Subst., Adj., Produkt: Verb

\section{Istzustand:}

(a) in beiden Wörterbüchern

(b) STERZ: nur die Grundvariante Ge-, die mit dem Partizippräfix ge- (gearmt, gefallen, geachtet) in einem Wörterbuchartikel behandelt wird; die Angabe zum Wegfall des -e fehlt;

DUDEN: wie oben dargestellt, nur die Angabe zum Wegfall -e fehlt;

(c) STERZ: Wortartenzugehörigkeit der Basis (Verb, Subst.) wie auch des Produktes (Subst., aber ohne Genusangabe) explizit verzeichnet, Bildungseinschränkungen nicht erwähnt

DUDEN: nur mit Verben als Basis, unter den Verben, die nicht als Basis dienen können, sind nicht genannt: reflexive Verben und Modalverben; die Wortartzugehörigkeit der Produkte nur implizit an der Genusangabe (das) bzw. an den Beispielen abzulesen

(d) STERZ: keine Angaben ${ }^{14}$ DUDEN: realisiert wie oben dargestellt

(e) STERZ: wie oben dargestellt, dazu aber noch die Angabe Adj. als Basis und Adv. als Produkt angeführt

DUDEN: keine Angaben zur Basis, Produkte als Adj. verzeichnet

(f) STERZ: als Basen Verben angeführt ("ve složeninách s $v$ "/"in Zusammensetzungen ${ }^{15}$ mit Verben"), Wortartenzugehörigkeit der Produkte nur an Beispielen abzulesen

DUDEN: Wortartenzugehörigkeit der möglichen Basen nicht erwähnt, als Produkte Verben angeführt

\subsubsection{Aussprache}

Zu erwarten: bei allen Affixen Angabe zur Betonung bzw. Vokallänge: Ge-...(-e) und entunbetont, -sam unter Nebenakzent (lang auszusprechen)

Istzustand: nur im STERZ bei ent- und -sam ${ }^{16}$

\subsubsection{Wortbildungsbedeutung, Äquivalenz und Beispiele}

\section{Zu erwarten:}

\footnotetext{
14 Das STERZ gibt in der Regel keine grammatischen Angaben an. Der Wörterbuchartikel -weise, in dem man erfahren kann, dass Adjektive und Adverbien auf -weise nicht gesteigert werden, ist eine Ausnahme.

15 Als Zusammensetzungen (slož. - "složenina") werden im STERZ "echte Zusammensetzungen" wie auch Derivate bezeichnet.

16 Ausspracheangabe fehlt aber z. B. bei los-, -los oder -ung.
} 
(a) bei allen Affixen verständliche Bedeutungserklärungen einzelner Lesarten (ggf. einschließlich Angaben zur wortbildungsmodellinternen und wortbildungsmodellexternen Konkurrenz, vgl. Ge- vs. Ge-...(-e), resp. Ge-...(-e) und -ei/-erei), ergänzt durch Überblick der häufigsten systemhaften Äquivalente im Tschechischen, und zwar obligatorisch bei sehr produktiven Modellen ${ }^{17}$ (d. h. bei Ge-...(-e) in der Verwendung als Nomen actionis und bei ent- in der Verwendung 'entfernen', 'rückgängig machen'), sowie durch morphologisch und semantisch durchsichtige Wortbildungsprodukte als Beispiele.

(b) $G e-\ldots(-e)$ :

A. fortwährender Prozess (oft lästig/unangenehm): im Tsch. v. a. Suffixe -ní, -ot: das Gebell :: štěkání, štěkot Anm.: Wo Formen mit und ohne -e nebeneinander stehen (Gebell-Gebelle), kann an die Form mit - e eine pejorative Konnotation gebunden sein

B. Produkt/Ergebnis: das Gewimmel :: hemžení, dav

C. Gesamtheit: im Tsch. oft das Suffix -oví oder Plural: das Geäst :: větvoví, větve (Äste) Gebein :: ostatky, kosti, Gebüsch :: křoví

(c) -sam:

A. potentiell-passivisch (was ge-X-t werden kann): im Tsch. verschieden Suffixe, v. a. -telný und -ný: einprägsam :: zapamatovatelný, biegsam :: ohebný

B. Neigung zu einer Handlung aufweisend: im Tsch. verschiedene Suffixe, z. B. -ný, -itý, ivý: mitteilsam :: hovorný, arbeitsam :: pracovitý, sparsam :: šetřivý

C. ornativ (X habend, von $\mathrm{X}$ erfüllt, $\mathrm{X}$ bereiten): im Tsch. verschiedene Suffixe, z. B. -ný, ivý, -ový: wirksam :: účinný, betriebsam :: snaživý, erholsam :: odpočinkový

(d) ent-

A. lokal-dynamisch: im Tsch. v. a. Präfixe, z. B. od-, z-: enteilen :: odchvátat/odkvapit, entschwinden :: zmizet

B. entfernen, rückgängig machen: im Tsch. v. a. Präfixe od-, vy-, de-: entfärben :: odbarvit, jn4 enterben :: vydědit někoho, entmilitarisieren :: demilitarizovat

C. inchoativ - Beginn von X: im Tsch. v. a. Präfixe roz-, vz-, za-: entfachen (das Feuer) :: rozdmýchat (oheň), entbrennen :: vzplát, entzünden :: zapálit

\section{Istzustand:}

(a) STERZ: knappe, zum Teil unvollständige oder allzu allgemeine, auf Tschechisch verfasste Kommentare zur Wortbildungsbedeutung: bei Ge-...(-e) Lesart 1 fehlt die Angabe [+dauernd +wiederholt] und oft [+lästig +unangenehm], bei -sam in der Lesart 1 ist die Bedeutungserklärung m. E. zu allgemein formuliert - cf. unter (c); jede Lesart mindestens mit einem Beispiel exemplifiziert ${ }^{18}$, wovon einige jedoch bereits isoliert sind (vgl. z. B. Gebirge Berg), in der Regel keine Äquivalenzangaben auf der Affixebene ${ }^{19}$, doch Übersetzungen

\footnotetext{
17 Die Angaben zur Produktivität nach dem WFDG.

${ }^{18}$ Keine Beispiele finden sich dagegen bei -lein und -los.

19 Von dieser Regel gibt es im STERZ mindestens zwei Abweichungen. Bei $u r-/ U r$ - ist das tschechische Präfix pra- als Äquivalent angeführt. Unter an- bei dem Beispielwort anfaulen ist im tschechischen Übersetzungsequivalent ("nahnívat") das Präfix ("na-") kursiv gedruckt (auch als Druckfehler erklärbar?).
} 
einzelner Beispiele konsequent angegeben, wodurch in einigen Fällen das Äquivalent auf der Affixebene eruierbar ist (vgl. -ní bei Geächze, Geacker: sténání, oráni); zum Teil anfechtbare Bedeutungsstrukturierung (cf. b-d); keine Angabe zur wortbildungsmodellinternen und externen Konkurrenz

DUDEN: teilweise sehr umfassende, auf Deutsch geschriebene Kommentare; zahlreiche Beispiele (lexikalisierte Wortschatzeinheiten wie auch Okkasionalismen); drastisch reduzierte Äquivalentangaben, die auch dort fehlen, wo relativ systemhafte Äquivalenzverhältnisse vorliegen (vgl. z. B. Ge-...(-e)); falls Übersetzung angeboten wird, steht sie am Artikelende und wird nicht auf einzelne Lesarten bezogen; Beispiele werden nicht übersetzt; teilweise eine zu detaillierte Lesartenstrukturierung - vgl. die Bedeutungsstrukturierung (Lesarten 1a-c) bei ent-, die von antonymischen Beziehungen ausgeht; Angaben nur zur wortbildungsmodellexternen Konkurrenz mit -ei/-erei

(b) STERZ: Im STERZ wird etymologisierend vorgegangen, indem das substantivische und das verbale Affix in einem gemeinsamen Wörterbucheintrag behandelt werden; dem substantivischen Modell sind die Lesarten 1 und 2 zuzurechnen (sie entsprechen den Lesarten A und C wie oben dargestellt); die Lesart B (Produkt, Ergebnis) fehlt.

DUDEN: nur die Lesart A (Nomina actionis)

(c) STERZ: 3 Lesarten: 1. die durch das Verb ausgedrückte Eigenschaft (ehrsam, sorgsam) entspricht der Lesart C. 2. Fähigkeit, Neigung (arbeitsam, biegsam) - entspricht der Lesart B, doch arbeitsam ist eher der Lesart A (potenziell-passivisch) zuzurechnen, die im STERZ aber nicht als selbständige Lesart geführt wird. 3. Ähnlichkeit: wundersam - einem Wunder ähnlich, das aber eher der Lesart 1 (ornativ) zuzuordnen ist (cf. Fleischer/Barz 2007: 266).

DUDEN: in modifizierter Reihenfolge (A, C, B) wie oben dargestellt

(d) STERZ: 4 Lesarten: 1. Beseitigen von etw. (entfärben) - entspricht der Lesart B 2. das Sichentfernen (entfallen, entspringen): entspricht der Lesart A 3. Entstehen, Anfang (entbrennen): entspricht der Lesart C 4. ins Gegenteil verkehren (entstellen): diese Lesart ließe sich eventuell auch der Lesart B zuordnen (Verben wie entstellen oder entarten sind stark idiomatisiert).

DUDEN: insgesamt in 10 (!) Lesarten ausdifferenziert ${ }^{20}$, die aber im Grunde genommen auf die drei Hauptlesarten wie oben dargestellt zurückführbar sind.

\subsubsection{Verweisstrukturen}

\section{Zu erwarten:}

(a) Ge-...(-e): A zum Teil informell und abwertend

(b) ent-: A und $\mathrm{C}$ in der Regel gehoben

\section{Istzustand:}

(a) nur im DUDEN Angabe "oft abwertend"

(b) keine Angaben im STERZ wie DUDEN 


\subsubsection{Produktivität}

\section{Zu erwarten:}

(a) $G e-\ldots(-e)$ : A sehr produktiv B und C nicht produktiv (b) -sam: nicht produktiv

(c) ent-: A und $\mathrm{C}$ nicht produktiv $\mathrm{B}$ sehr produktiv

Istzustand: in beiden Wörterbüchern keine Angaben.

\subsection{Fazit}

In welchem der hier analysierten Wörterbücher die bessere Bearbeitung der Affixe vorliegt, ist schwer $\mathrm{zu}$ entscheiden. Das STERZ irritiert durch zahlreiche Auslassungen und Inkonsequenzen sowohl im Bereich der Makro- als auch der Mikrostruktur, der DUDEN durch dürftiges, in einem Übersetzungswörterbuch kaum akzeptables Äquivalentangebot und zum Teil unübersichtliche Artikelstruktur. Obwohl das Angebot an Affixangaben in beiden Wörterbüchern lückenhaft und unsystematisch ist, ist hier eine gewisse Nachsicht vielleicht berechtigt, denn andere deutsch-tschechische Wörterbücher bieten in dieser Hinsicht noch erheblich weniger an, indem sie auf die Inventarisierung von Wortbildungselementen völlig verzichten.

Im folgenden Kapitel ist darauf einzugehen, wie die Affixe im GAWDT, einem neuen deutsch-tschechischen Wörterbuch im Entstehen, gehandhabt werden.

\section{Affixe im GAWDT am Beispiel des Präfixes/Zirkumfixes Ge-...(-e)}

\subsection{Zum Projekt des GAWDT}

Das GAWDT ist primär als eine elektronische, wortartenspezifisch angelegte Datenbank konzipiert. ${ }^{21}$ Die Adressatengruppe des GAWDT sind v. a. tschechische Muttersprachler (Übersetzer, Akademiker, Germanisten, Deutschlerner), denen keine umfassende und aktuelle, kontrastiv ausgerichtete Grammatik des Deutschen zur Verfügung steht, im Rahmen derer die Wortbildung mit behandelt werden könnte (die Wortbildung des Deutschen wird weder in Povejšil (1987) noch in Štícha (2003) explizit erfasst). In das GAWDT sollen Wörterbuchartikel ausgewählter Wortbildungselemente sowie eine ausführliche Wörterbuchgrammatik aufgenommen werden. Einen festen Bestandteil der lexikographischen Arbeiten stellen Forschungen dar, die auf kontrastive Analysen des InterCorp-Parallelkorpus Deutsch-Tschechisch (www.intercorp.cz) abzielen. Das Wörterbuch bzw. die begleitende Forschung fußt auf modernen korpusanalytischen Methoden, die im Rahmen der Kooperation mit dem Forschungsbereich Korpuslinguistik am Institut für Deutsche Sprache in Mannheim (www.ids-mannheim.de/kl/projekte/methoden/) getestet werden. Der systemorientierte Blick,

\footnotetext{
20 Solche detaillierten Angaben sind für philologisch Interessierte sicher nützlich, doch unerfahrene Wörterbuchbenutzer mit eingeschränkten Deutschkenntnissen werden sie eher entmutigen.

${ }^{21}$ Die ursprüngliche Software ist von Dipl.-Ing. Pavel Kopřiva entwickelt worden. Seit 2010 wird der Datenbankinput schrittweise auf eine neue Software für eine online-Datenbank von Dipl.-Ing. Karel Klouda, Ph.D. umgestellt. Die adjektivische Subdatenbank des GAWDT ist im März 2013 den Studierenden und Lehrenden der Philosophischen Fakultät der Karls-Universität in Prag zur Verfügung gestellt worden (siehe unter lexarchiv.ff.cuni.cz/slovnik/).
} 
der eine Verallgemeinerung bedingt, und die verwendungsorientierte Auffassung, beide im Dienste einer funktionalen Sprachbeschreibung, begegnen einander.

\subsection{Ein Wörterbucheintrag für das Präfix/Zirkumfix Ge-...(-e) entsteht}

"Die konzentrierte Bearbeitung eines Wortbildungsmodells bzw. -typs kann einer besseren Erfassung einer übersichtlichen Anzahl von Wortbildungsbedeutungen und den Reflexionen bei der Äquivalenzsuche gut tun, zumal individuelle Züge der Lexeme (Bedeutungsnuancen) ein besonderes Anliegen des Adressatenkreises verkörpern" (Vachková 2007: 58). Wörterbucheinträge der Wortbildungselemente, die in einer speziellen Subdatenbank des GAWDT abgespeichert werden, entstehen in fünf Schritten:

(a) Entscheidung der Redaktion, ein Wortbildungselement im GAWDT zu inventarisieren;

(b) Bestandaufnahme entsprechender Wortbildungsprodukte und

(c) deren detailierte Analyse - Sichtung des Korpusmaterials und Bearbeitung der Wörterbuchartikel einzelner Lexeme (cf. Anhang IV);

(d) Verallgemeinerung und Synthese unter Berücksichtigung der verfügbaren Beschreibungen jeweiliger Wortbildungsmodelle - Erarbeitung des Wörterbucheintrags eines Wortbildungselementes;

(e) Verlinkungen von einzelnen Wortbildungsmodellen mit dazugehörigen Wortbildungsprodukten.

Ad (a):

Die Redaktion hält sich bei der Auswahl der zu inventarisierenden Wortbildungselemente im Allgemeinen an dem Quellenwörterbuch DUW, das wegen seines makrostrukturellen Umfangs als Orientierungshilfe eine wichtige Rolle spielt ${ }^{22}$, und am D-BWB. Besonders detailliert werden vorwiegend (doch nicht ausschließlich) die produktiven Wortbildungsmodelle (siehe die Wörterbuchartikel von $G e-\ldots(-e)$ und $G e-\ldots$ unten) bearbeitet.

Ad (b):

Die Aufnahme von individuellen Wortbildungsprodukten, welche einem Wortbildungsmodell zuzuordnen sind und welche die Grundlage für die Erarbeitung von entsprechenden Wörterbucheinträgen der Wortbildungselemente darstellen, ist jedoch - von korpuslinguistischen Analysen ausgehend - zu relativieren (cf. Vachková 2006: 170). Die Bestandaufnahme einzelner Lexeme erfolgt in der Regel unter Berücksichtigung des DUW, der $\mathrm{CCDB}^{23}$, der DeReWo-Listen ${ }^{24}$ und DeReKo ${ }^{25}$. Im Falle der Neutra auf Ge-...(-e) handelt es sich um knapp 500 Einheiten ${ }^{26}$ (einschließlich idiomatischer Bildungen, siehe Anhang II).

\footnotetext{
22 Im Rahmen der Arbeiten am GAWDT werden neben den bereits erwähnten Wörterbüchern auch andere sekundäre Quellen herangezogen, deren vollständige Liste an dieser Stelle nicht wiedergegeben werden kann. Genannt seien hier deswegen nur das DWDS, DTWP und D-R.

23 Belica, C.: Kookkurrenzdatenbank CCDB. Eine korpuslinguistische Denk- und Experimentierplattform für die Erforschung und theoretische Begründung von systemisch-strukturellen Eigenschaften von Kohäsionsrelationen zwischen den Konstituenten des Sprachgebrauchs. (C) 2001-2007 Institut für Deutsche Sprache, Mannheim. In der CCDB sind 220309 verschiedene Kookkurrenzprofile gespeichert (Stand: April
} 
Ad (c):

Die Unterlage für die Mikrostruktur bildet ein segmentierter Datenbankinput (zu Details am Beispiel der adjektivischen Subdatenbank siehe Vachková 2011). Im Vordergrund steht die Darstellung der Kollokabilität, sowie die Erfassung von Paradigmatik und Syntagmatik, die auf der Basis der Kookkurrenzanalyse und der auf deren Grundlage enwickelten korpuslinguistischen Tools erfolgt. Die Datenbankoutputs verfügen über eine Mikrostruktur mit Angaben zur Rechtschreibung, mit Ausspracheangaben, grammatischen Informationen einschließlich Varianz, syntaktischen Strukturen, Phrasemen, Sprichwörtern in Auswahl etc. Eine bedeutende Rolle spielt das Parallelkorpus InterCorp ${ }^{27}$ - auch in Bezug auf die Erfassung der Äquivalenz unterhalb der Wortebene (s. Anhang III). Erforschung lokaler Verwendungskontexte bildet hierbei einen integrierten Bestandteil der praktischen lexikographischen Arbeit am GAWDT. Individuelle Leistungen der Übersetzer können berücksichtigt und auch unter wortbildungsanalytischem Aspekt ausgewertet werden. Eine Auswertung von Äquivalenten der im InterCorp vorgefundenen Nomina actionis auf Ge-...(e) hat z. B. ergeben, dass bei etwa mehr als 50\%, resp. $20 \%$ dieser Wortbildungen tschechische Entsprechungen auf -ní-ení, resp. -ot ausgemacht werden können. Diese Wortbildungselemente konnten also als zentrale tschechische Äquivalente des deutschen Präfixes/Zirkumfixes Ge-...(-e) (Nomina actionis) identifiziert werden. ${ }^{28}$

$\operatorname{Ad}(d)$ :

Der auf Grund der bisher von der GAWDT-Redaktion durchgeführten Analysen des Wortbildungselementes sowie dessen bestehender Beschreibungen (Fleischer/Barz 2007; Donalies 2005; Wellmann 1975 u. a. m. und Wörterbucheinträge des Modells im LGDaF, DUW, WDW, STERZ und DUDEN) erarbeitete Eintrag hat aktuell (Stand: April 2013) folgende Gestalt ${ }^{29}$ :

${ }^{1}$ Ge-...(-e)

-ge-...-e (das Herumgehopse) seltener, in der Regel nur A

2013), sodass sie als ein relativ verlässliches Korrektiv bzw. eine relativ verlässliche Basis für den Lemmabestand eines Wörterbuchs aufgefasst werden kann, das etwa 130000 Einträge enthält (DUW).

${ }^{24}$ Korpusbasierte Wort-/Grundformenlisten DeReWo (Der Deutsche Referenzwortschatz), frei verfügbar unter www.ids-mannheim.de/kl/derewo/. Es handelt sich um mehrere, auf der Grundlage des DeReKo (Das Deutsche Referenzkorpus) erstellte Häufigkeitslisten von im deutschen Sprachgebrauch am häufigsten vorkommenden Grund- bzw. Wortformen.

25 Das Deutsche Referenzkorpus DeReKo (www.ids-mannheim.de/k1/projekte/korpora/), am Institut für Deutsche Sprache, Mannheim. Das Korpus enthält 6,1 Milliarden Textwörter (Stand: März 2013).

26 Die Variante ...-ge-...-e ist hier nicht berücksichtigt, weil die Ermittlung entsprechender Wortbildungsprodukte eine manuelle Sichtung des immensen Korpusmaterials voraussetzen würde, welche die Arbeit am GAWDT im Hinblick auf die Anzahl der zu bearbeitenden Wortbildungselemente lähmen würde. Auch hier zeigt sich, dass die Darstellung der Wortbildung in einem Wörterbuch stets die Kunst des Möglichen ist.

27 InterCorp, Parallelkorpora der Philosophischen Fakultät der Karls-Universität Prag (cf. unter ucnk.ff.cuni.cz/intercorp/). Das Korpus umfasst v. a. deutsche und tschechische belletristische und journalistische Texte. Das Korpus wird kontinuierlich ausgebaut, wobei das deutsche Subkorpus aktuell mehr als ${ }^{37}$ Millionen Textwörter enthält (Stand: April 2013).

${ }^{28}$ Nicht alle im InterCorp vorgefundenen Entsprechungen eines deutschen Lexems fließen, u. a. wegen deren starker kontextueller Abhängigkeit, als Übersetzungsäquivalente in den entsprechenden Wörterbuchartikel des GAWDT ein, doch sie können unter wortbildungsanalytischem Aspekt relevante Informationen liefern und sind somit zu berücksichtigen.

${ }^{29}$ Für den Zweck dieses Beitrags ist der Wörterbuchartikel ins Deutsche übersetzt worden, in der Datenbank des GAWDT ist eine Version mit tschechischer Metasprache abgespeichert. 
Ge-... Präfix
Ge-...-e/-ge-...-e Zirkumfix

Subst., Neutra (das) einige wenige Maskulina wie der Geruch oder der Gesang sind isolierte Relikte aus älteren Entwicklungsphasen des Deutschen

unbetont

A

im Tschechischen in der Regel Suffigierung

-ní/-ení Suffix, Neutra: das Gebrüll(e) řvaní, výkřriky, řev (brüllen - řvát), das Gehämmer(e) bušení (hämmern - bušit), das Gebimmel(e) zvonění (bimmeln - zvonit)

-ot Suffix, Maskulina: das Gebell(e) štěkot, štěkání (bellen - štěkat), das Gekicher(e) chichot (kichern -

chichotat se, hihňat se), das Gewisper(e) šepot (wispern - šeptat)

-ba Suffix, Feminina seltener: das Geknall(e) střelba, palba (knallen - stř́let, pálit)

-nice Suffix, Feminina seltener: das Gerangel(e) tahanice (rangeln - tahat se s někým o něco)

sehr produktiv

zum Teil abwertend und informell

Verwendet, um aus einem Verb ein Substantiv zu machen, das einen (nicht immer, doch oft lästigen, unangenehmen, zu lange dauernden) Prozess bezeichnet: das Gebrüll(e) (otravný) řev/(neustálé) řvaní - brüllen řvát; mit dem Suffix - $e$ kann manchmal eine stärkere negative Konnotation verbunden sein: das Gebelle (velmi) otravné/(v)otravný/obtěžující/neustálé/neustálý/věčný štěkání. Die Verwendung des Suffixes -e können daneben Faktoren wie Satzrhythmus und Reim beeinflussen bzw. formale Angleichung an kumulierte lexikalische Einheiten in der Umgebung des betreffenden $G e-\ldots(-e)$-Derivats. Das Vorkommen des Endungs-e kann stärker die Nomina actionis im Gegensatz zu Lesarten B und C kennzeichnen (das Gebete vs. das Gebet).

SYNONYME $\uparrow$-(er)ei das Getanze/die Tanzerei - (neustálé/ý, neobratné/ý) tancování/tanec/křepčení Formen auf Ge-...(-e) werden in der Regel bevorzugt, wenn (a) die Sprecher/Schreiber die als störend oder unangemessen ausgeführt empfundene Tätigkeit anderer kommentieren, (b) die Sprecher/Schreiber direkt dem Geschehen aktuell beiwohnen bzw. ihm ausgesetzt sind (c) die Sprecher/Schreiber die Tätigkeit nicht als habituell ausgeführt empfinden, (d) man eine mehr globale, numerusindifferente Bezeichnung fokussiert, (e) eher Überdruss als Missbilligung oder Tadel zum Ausdruck gebracht werden soll, (f) sich die Basis auf Witterungserscheinungen bezieht (donnern - das Gedonner).

substantivierter Infinitiv das Gebell/das Bellen - štěkot, štěkání

Bei dem substantivierten Infinitiv fehlt in der Regel der Aspekt des Störenden (falls dieser bereits nicht in dem Basisverb enthalten ist), der für die Wortbildungsprodukte auf $G e-\ldots(-e)$ dagegen oft typisch ist. So ist z. B. das Trommeln kontextuell mit der Musik verbunden, während das Getrommel(e) in ähnlichen Kontexten verwendet wird wie Bezeichnungen für andere als unangenehm und störend empfundene Geräusche.

als Basis nicht möglich: Hilfs-, Reflexiv- und Modalverben, Verben auf -ieren und Verben mit untrennbaren Präfixen; trennbare Präfixverben und verbale Komposita als Basis sind jedoch möglich: das Mitgeklatsche mitklatschen, das Herumgehopse - herumhopsen, das Gefrühstücke - frühstücken. Im Falle von Partikelverben als Basis wird Ge- zwischen die Partikel und den Stamm geschoben (das Mitgeklatsche, das Herumgehopse).

Es gibt relativ zahlreiche Überlappungen zwischen Lesart A und Lesart B, vgl. z. B. das Gequatsche, das sowohl als blábolení (Prozess) als auch als blábol (Ergebniss) übersetzt werden kann.

B

im Tschechischen v. a. verschiedene Suffixe, z. B.:

-nice Suffix, Feminina: das Gekritzel čmáranice (kritzeln - čmárat) das Gedränge tlačenice, mačkanice (sich drängen - tlačit se, mačkat se)

-ice Suffix, Feminina: das Gemisch směsice (mischen - (s)míchat)

-ina Suffix, Feminina: das Gemisch smíšenina (mischen - (s)míchat)

-ba Suffix, Feminina: das Gebet modlitba (beten - modlit se) 
nicht produktiv

bezeichnet ein Produkt, ein Ergebnis, etwas, das durch die genannte Handlung entstanden ist

SYNONYME $\uparrow$-(er)ei das Gekritzel/die Kritzelei - čmáranice, $\uparrow$-ung das Gemisch/die Mischung - směs

Es gibt zahlreiche Überlappungen zwischen Lesart A und Lesart B, vgl. z. B. das Gequatsche, das sowohl als blábolení (Prozess) als auch als blábol (Ergebniss) übersetzt werden kann.

C systemhafte Äquivalenz unterhalb der Wortebene eher problematisch auszumachen, es handelt sich um verschiedene Suffixe, z. B.

-dlo/-idlo Suffix, Neutra: das Gebläse dmychadlo (blasen - foukat, dmýchat)

nicht produktiv

bezeichnet entweder einen Gegenstand, mit dem Tätigkeiten ausgeführt werden, oder etwas, mit dessen Hilfe man etwas tun oder erreichen kann

${ }^{2} \mathrm{Ge}-\ldots(-\mathrm{e})$

Ge-... Präfix

Ge-...-e Zirkumfix seltener

Subst., Neutra (das) es gibt einige wenige Maskulina und Pluralia tantum (beides Personenbezeichnungen) wie der Geselle, der Gefährte, die Gebrüder, von denen die meisten isoliert bzw. idiomatisiert sind

unbetont

im Tschechischen in der Regel Plural oder Suffigierung

-oví Suffix, Neutra: das Geäder žilkoví, žilky, žíly (die Ader - žíla)

-stvo Suffix, Neutra: das Gebirge horstvo, hory (der Berg - hora)

-ctvo Suffix, Neutra: das Getier zviŕrectvo, živočichové (das Tier - zvíre)

-na Suffix, Feminina: das Gebüsch křovina, houština (der Busch - keř)

-isko Suffix, Neutra: das Gebüsch křovisko (der Busch - keř)

nicht produktiv

Das Modell ergibt Kollektiva; es bezeichnet eine Gruppe von Sachen, Tieren u. Ä.: das Gebein - kosti, das Gedärm - střeva, das Getier - zviŕrena, zvíriectvo. Wortbildungsprodukte mit dem Endungs-e sind selten und in der Regel gehoben bzw. veraltet (das Geäste, das Gebüsche, das Gesträuche); bei einigen, meist mehr oder weniger idiomatisierten Wortbildungen ist dagegen das Endungs-e der Normalfall (das Gebirge, das Gelände, das Gemüse).

\section{SYNONYME}

$\uparrow$-werk das Gebüsch/das Buschwerk - křoví, křovina

Nicht immer sind entsprechende Wortbildungsprodukte kontextuell völlig austauschbar - so wird z. B. das Gebüsch viel häufiger als das Buschwerk auch als Bezeichnung für Tat- oder Fundort verwendet, während das Buschwerk eher nur im Ökologiediskurs vorkommt. Bei einigen Lexempaaren erscheint das meist seltenere werk-Derivat verstärkt in solchen Kontexten, in denen nicht nur allgemein eine Gesamtheit von etwas bezeichnet werden soll, sondern in denen eine Gesamtheit von Entitäten in deren ausgeprägterer räumlicher Dimensionalität fokussiert wird.

Plural der Wortbildungsbasis das Gezweig/die Zweige - větvoví, větve 
substantivische Basis in der Regel mit einer Vokaländerung: das Geäder - die Ader, das Gebirge - der Berg Ad (e):

In Wörterbuchartikeln der einschlägigen Wortbildungsprodukte wird auf die jeweilige Lesart des Wortbildungselementes mittels eines Hyperlinks (Verweissymbol $\uparrow$ ) verwiesen. Erschließung unbekannter Lexeme kann somit sowohl mithilfe abstrakter Baumuster als auch individueller Wortanalogie erfolgen. Dies entspricht im Wesentlichen den Vorschlägen, die im Zusammenhang mit der lexikographischen Behandlung der Affixe in Augst (2005: 122) formuliert worden sind.

\section{$4 \quad$ Zusammenfassung und Ausblick}

Die moderne Lexikographie stellt eine eigenständige Disziplin dar, der eigene Theorie zugrunde liegt, wobei die Lexikographen alltäglich konkrete Entscheidungen zu treffen haben, welche mit linguistischen Fragestellungen oft nur sehr lose verknüpft sind, was sich z. B. auch im Bereich der lexikographischen Erfassung von Wortbildung zeigt. Im Zusammenhang mit der deutsch-tschechischen Lexikographie kann festgehalten werden, dass sich die bestehenden deutsch-tschechischen Wörterbücher (auch) hinsichtlich der Darstellung der Wortbildungselemente in einem suboptimalen Zustand befinden. Die hier selbstverständlich nur auswahlsweise behandelten Defizite sind abzubauen, indem in der deutsch-tschechischen Lexikographie ein grundlegender Paradigmenwechsel eingeleitet und der Wörterbuchbenutzer mit seinen Bedürfnissen in Vordergrund gerückt wird, wie es im Falle des GAWDT geschieht. Große elektronische Korpora fordern dabei auch im Bereich der Wortbildung zu empirischen Materialanalysen auf, die in der Vergangenheit aus technischen Gründen nicht möglich waren.

\section{Danksagung}

Für wertvolle Kommentare zu früheren Versionen dieses Beitrags möchte ich mich herzlich bedanken bei (in alphabetischer Reihenfolge) Prof. Dr. Irmhild Barz (Universität Leipzig), Dr. Hannelore Poethe (Universität Leipzig) und meiner Doktormutter Doc. PhDr. Marie Vachková, Ph.D. (Karls-Universität Prag) sowie den beiden anonymen Gutachtern von Linguistik online. Mein aufrichtiger Dank gehört auch dem DAAD für die Unterstützung während meines Stipendiumaufenthalts in Leipzig (Wintersemester 2010/2011), während dessen dieser Text in seinen Grundzügen konzipiert worden ist.

\section{Literatur}

\section{(a) Wörterbücher}

D-BWB = Müller, Wolfgang $\left({ }^{2} 1985\right)$ : Duden - Bedeutungswörterbuch. Mannheim: Bibliographisches Institut.

D-R = Dudenredaktion (Hrsg.) ( $\left.{ }^{3} 2008\right)$ : Duden - Redewendungen. Mannheim etc.: Dudenverlag.

DTWP = Heřman, Karel et al. (2010): Deutsch-tschechisches Wörterbuch der Phraseologismen und festgeprägten Wendungen. Prag: C. H. Beck. 
DUDEN = Müller, Wolfgang et al. (1993): Duden: německý výkladový slovník s českými ekvivalenty (Duden: Deutsches Wörterbuch mit tschechischen Äquivalenten, tschechische Äquivalente von Hartl, Miroslav/Křivská, Jaroslava/Raiterová, Hana). Praha: Mladá fronta.

DUW $=\left({ }^{4} 2001\right)$ Duden - Deutsches Universalwörterbuch. Mannheim etc.: Dudenverlag. [CD-ROM]

DUW-P = (2001) Duden - Deutsches Universalwörterbuch. 4. Aufl., Mannheim etc.: Dudenverlag.

DWDS = Das Digitale Wörterbuch der deutschen Sprache. Berlin-Brandenburgische Akademie der Wissenschaften. www.dwds.de/, Stand: 25.08.2013).

LGDaF = Götz, Dieter/Haensch, Günther/Wellman, Hans (eds.) (1999): Langenscheidts Großwörterbuch Deutsch als Fremdsprache. Berlin etc.: Langenscheidt. [CD-ROM]

LIN = Lingea (2006): Německo-český, česko-německý velký slovník: nejen pro prekladatele (Großes Wörterbuch deutsch-tschechisch, tschechisch-deutsch: nicht nur für Übersetzer). Brno: Lingea.

OALD = Wehmeier, Sally et al. (eds.). ("2005): Oxford Advanced Lerner's Dictionary. Oxford: Oxford University Press.

SIEB = Siebenschein, Hugo et al. ( $\left.{ }^{2} 2006\right)$ : Německo-český slovník (Deutsch-tschechisches Wörterbuch). 2 Bände. Voznice: Leda.

STERZ = Sterzinger, Josef Václav (1916-1935): Encyklopedický německo-český slovník (Enzyklopädisches Deutsch-böhmisches Wörterbuch). 4 Bände. Prag: J. Otto (digitalisierte Version verfügbar unter german.ff.cuni.cz/?q=node/184).

WDW $=(1997)$ Wahrig - Deutsches Wörterbuch. Gütersloh/München: Bertelsmann Lexikon Verlag. [CD-ROM]

WFDG = Augst, Gerhard (2009): Wortfamilienwörterbuch der deutschen Gegenwartssprache (Studienausgabe). Tübingen: Max Niemeyer Verlag.

\section{(b) Forschungsliteratur}

Ascoop, Kristin (2005): "Affixoidhungrig? Skitba! Status und Gebrauch von Affixoiden im Deutschen und Schwedischen". Germanistische Mitteilungen 62: 17-28.

Augst, Gerhard (2005): "Die Wortfamilie als Ordnungskriterium in elementaren Wörterbüchern". In: Fix, Ulla et al. (eds.) (2005): Zwischen Lexikon und Text. Lexikalische, stilistische und textlinguistische Aspekte. Stuttgart, Hirzel: 111-124 (= Abhandlungen der Sächsischen Akademie der Wissenschaften zu Leipzig. Philologischhistorische Klasse 78/4).

Barz, Irmhild (2000): "Zum heutigen Erkenntnisinteresse germanistischer Wortbildungsforschung. Ein exemplarischer Bericht". In: Barz, Irmhild/Schröder, Marianne/Fix, Ulla (eds.) (2000): Praxis- und Integrationsfelder der Wortbildungsforschung. Heidelberg, Winter: 299-316.

Barz, Irmhild (2001): "Wortbildungsbeziehungen im einsprachigen Bedeutungswörterbuch". In: Korhonen, Jarmo (ed.) (2001): Von der mono- zur bilingualen Lexikographie für das Deutsche. Frankfurt am Main etc., Peter Lang: 85-100 (=Finnische Beiträge zur Germanistik 6).

Barz, Irmhild (2002): "Die Wortbildungsmittel im de Gruyter Wörterbuch Deutsch als Fremdsprache". In: Wiegand, Herbert Ernst (ed.) (2002): Perspektiven der pädagogischen Lexikographie des Deutschen II. Untersuchungen anhand des "de Gruyter Wörterbuchs Deutsch als Fremdsprache". Tübingen, Max Niemeyer: 105-121 (=Lexicographica Series Maior 110).

Barz, Irmhild (2003): "Affixe im GWDS". In: Wiegand, Herbert Ernst (ed.) (2003): Untersuchungen zur kommerziellen Lexikographie der deutschen Gegenwartssprache I. Tübingen, Max Niemeyer: 383-393 (= Lexicographica. Series Maior 113). 
Bergenholtz, Henning (2000): "Lexikographie und Wortbildungsforschung". In: Barz, Irmhild/Schröder, Marianne/Fix, Ulla (eds.) (2000): Praxis- und Integrationsfelder der Wortbildungsforschung. Heidelberg, Winter: 19-30.

Brdar-Szabó, Rita (1996): "Aspekte der Wortbildung in der zweisprachigen Lexikographie unter besonderer Berücksichtigung eines neuen deutsch-ungarischen Handwörterbuchs". In: Hessky, Regina (ed.) (1996): Lexikographie zwischen Theorie und Praxis. Das deutschungarische Wörterbuchprojekt. Tübingen, Max Niemeyer: 75-98 (= Lexicographica. Series Maior 71).

Burgschmidt, Ernst (2004): "Theorie der Wortbildung und ihr Reflex im Wörterbuch". In: Herbst, Thomas et al. (eds.) (2004): Lexikographie, ihre Basis- und Nachbarwissenschaften. Tübingen: Max Niemeyer: 175-184 (= Lexicographica. Series Maior 118).

Bußmann, Hadumod (ed.) (2008): Lexikon der Sprachwissenschaft. Stuttgart: Kröner.

Čermák, František (1995): "Překladová lexikografie". In: Čermák, František/Blatná, Renata (eds.) (1995): Manuál lexikografie. Praha, H\&H: 116-136.

Čermák, František (2010): Lexikon a sémantika (Lexikon und Semantik). Praha: Nakladatelství Lidové noviny.

Donalies, Elke (2005): Die Wortbildung des Deutschen. Ein Überblick. Tübingen: Narr. (= Studien zur deutschen Sprache 27).

Eisenberg, Peter (1998): Grundriß der deutschen Grammatik. Das Wort. Stuttgart/Weimar: Metzler.

Elsen, Hilke (2009): "Affixoide: Nur was benannt wird, kann auch verstanden werden". Deutsche Sprache 37: 316-333.

Engelberg, Stefan/Lemnitzer, Lothar $\left({ }^{4} 2009\right)$ : Lexikographie und Wörterbuchbenutzung. Tübingen: Stauffenburg Verlag.

Fandrych, Christian (2011): "Wie geht es eigentlich den "Halbsuffixen"?" Deutsche Sprache 2/39: 137-153.

Fleischer, Wolfgang/Barz, Irmhild ( $\left.{ }^{4} 2007\right)$ : Wortbildung der deutschen Gegenwartssprache. Tübingen: Niemeyer.

Hansen, Sabine/Hartmann, Peter (1991): Zur Abgrenzung von Komposition und Derivation. Trier: Wissenschaftlicher Verlag. (= Fokus: linguistisch-philologische Studien).

Hyvärinen, Irma (2000): "Zur Wortbildung in einem deutsch-finnischen Großwörterbuch im Werden - Probleme der Lemmatisierung von Wortbildungsmitteln". In: Barz, Irmhild/Schröder, Marianne/Fix, Ulla (eds.) (2000): Praxis- und Integrationsfelder der Wortbildungsforschung. Heidelberg, Winter: 31-54.

Lüdeling, Anke/Evert, Stefan (2003): "Linguistic experience and productivity corpus evidence for fine-grained distinctions". In: Proceedings of the 2003 Corpus Linguistics Conference. Lancaster. www.linguistik.hu-berlin.de/institut/professuren/ korpuslinguistik/mitarbeiter-innen-en/anke/pdf/LuedelingEvert03.pdf, Stand: 25.08.2013)

Müller, Wolfgang (1989): "Die Beschreibung von Affixen und Affixoiden im allgemeinen einsprachigen Wörterbuch". In: Wiegand, Herbert Ernst et al. (eds.) (1989): Wörterbücher. Ein internationales Handbuch zur Lexikographie. Berlin/New York, de Gruyter: 869-882.

Mugdan, Joachim (1984): "Grammatik im Wörterbuch: Wortbildung". In: Herbert Ernst Wiegand (ed.) (1984): Studien zur neuhochdeutschen Lexikographie V. Hildesheim etc., Olms: 237-308.

Poethe, Hannelore (1996): "Wortbildung im Großwörterbuch Deutsch als Fremdsprache". In: Barz, Irmhild/Schröder, Marianne (eds.) (1996): Das Lernerwörterbuch Deutsch als Fremdsprache in der Diskussion. Sprache - Literatur und Geschichte. Heidelberg, Universitätsverlag C. Winter: 189-207 (= Studien zur Linguistik, Germanistik 12).

Povejšil, Jaromír (1987): Mluvnice současné němčiny (Grammatik des Gegenwartsdeutschen). Praha: Academia. 
Prćić, Tvrtko (1999): "The Treatment of Affixes in the 'Big Four' EFL Dictionaries". International Journal of Lexicography 4/12: 263-279.

Prćić, Tvrtko (2008): "Suffixes vs Final Combining Forms in English: A Lexicographic Perspective". International Journal of Lexicography 1/21: 1-22.

Schmidt, Günther Dietrich (1987): "Das Affixoid. Zur Notwendigkeit und Brauchbarkeit eines beliebten Zwischenbegriffs der Wortbildung". In: Wimmer, Rainer/Zifonun, Gisela (eds.) (1987): Deutsche Lehnwortbildung. Tübingen, Narr: 53-101 (= Forschungsberichte des IdS 64).

Schmidt, Marek/Vachková, Marie (2009): "Angaben zur deutschen Standardausprache im entstehenden Großen deutsch-tschechischen akademischen Wörterbuch (GDTAW) als Kunst des Möglichen". Aussiger Beiträge 3: 25-39.

Skála, Emil (1994): "Tschechisch-deutsche Sprachkontakte". Germanistica Pragensia XII. Prag, Karls-Universität: 7-22 (= AUC Philologica 2/1994).

Šemelík, Martin (2012): "Nestbildung in einem Übersetzungswörterbuch". Germanistica Pragensia XXII. Prag, Karls-Universität: 133-142 (= AUC Philologica 2/2012).

Šimečková, Alena (1987): "Zum Informationsangebot deutsch-tschechischer Wörterbücher". brücken. Germanistisches Jahrbuch DDR-ČSSR 1986/1987: 223-233.

Šimečková, Alena (1994): Untersuchungen zum trennbaren Verb im Deutschen I. Prag: Karls-Universität (= Acta Universitatis Carolinae, Philologica, Monographia CXIX).

Šimečková, Alena (1996): "Zur Modifikationsfunktion des Präverbs im deutschen komplexen Verb (am Material der NACH- und VOR-Verben)". In: Šimečková, Alena/Vachková, Marie (eds.) (1996): Wortbildung. Theorie und Anwendung. Prag, Karolinum-Verlag: 147154.

Štícha, František (2003): Česko-německá srovnávací gramatika (Tschechisch-deutsche vergleichende Grammatik). Praha: Argo.

Tarp, Sven (1995): "Wörterbuchfunktionen: Utopische und realistische Vorschläge für die bilinguale Lexikographie". In: Wiegand, Herbert Ernst (ed.) (1995): Studien zur bilingualen Lexikographie mit Deutsch II. Hindesheim/New York, Olms: 17-62.

Tarp, Sven (2009): "Beyond Lexicography: New Visions and Challenges in the Information Age". In: Bergenholtz, Henning/Nielsen, Sandro/Tarp, Sven (eds.) (2009): Lexicography at a Crossroads. Bern, Peter Lang: 17-32 (= Dictionaries and Encyclopedias Today, Lexicographical Tools Tomorrow).

Thurmair, Maria (1996): "Verbwortbildung und Verbklammer im Deutschen". In: Šimečková, Alena/Vachková, Marie (eds.) (1996): Wortbildung. Theorie und Anwendung. Prag, Karolinum-Verlag: 163-173.

Vachková, Marie (2002): "Wortbildung und zweisprachiges Wörterbuch." In: Barz, Irmhild/Fix, Ulla/Lerchner, Gotthard (eds.) (2002): Das Wort in Text und Wörterbuch. Leipzig/Stuttgart, Hirzel: 119-126 (= Abhandlungen der Sächsischen Akademie der Wissenschaften zu Leipzig, Philologisch-Historische Klasse 76/4).

Vachková, Marie (2006): "Korpusanalytische Methoden und Äquivalenz (am Beispiel der reihenbildenden Zweitglieder im adjektivischen Bereich)". In: Heinemann, Margot (ed.) (2006): Deutsch - Sprache der Euroregion(en): Konferenzbeiträge der internationalen Tagung an der Technischen Universität in Liberec am 22.-23. November 2006. Liberec, Nakladatelství Bor: 169-175.

Vachková, Marie (2007): "Adjektive auf -bar in kontrastiver und korpuslinguistischer Sicht. Eine Metalexikographische Betrachtung". Linguistica Pragensia 2/XVII: 57-74.

Vachková, Marie (2011): Das große akademische Wörterbuch Deutsch-Tschechisch: Ein erster Werkstattbericht. Frankfurt am Main etc.: Peter Lang.

Wellmann, Hans (1975): Deutsche Wortbildung. Zweiter Hauptteil: Das Substantiv. Düsseldorf: Pädagogischer Verlag Schwann (= Sprache der Gegenwart. Schriften des Instituts für deutsche Sprache in Mannheim XXXII). 
Wellmann, Hans ( $\left.{ }^{5} 1995\right)$ : "Die Wortbildung". In: Duden. Grammatik der deutschen

Gegenwartssprache. Mannheim etc., Dudenverlag: 399-539.

\section{(c) Internetquellen}

www.ids-mannheim.de, Stand: 25.08.2013.

www.wortwarte.de/index.html, Stand: 25.08.2013.

ucnk.ff.cuni.cz/intercorp, Stand: 25.08.2013.

www.dwds.de, Stand: 25.08.2013.

www.intercorp.cz, Stand: 25.08.2013.

Anhang I: STERZ und DUDEN - Wörterbuchartikel von Ge-...(-e), -sam und ent-

(a) $G e-\ldots(-e)$

(Ge $e_{\ldots+\ldots}, \mathfrak{g c}=\ldots+1$. předpona tvořicí substantiva od sloves: (5reädzze (od ädjzent) stenání; (5es acfer (oa adferti) orání. 2. tvoŕíi jména hromadná z podstatných: (5ebeirt (od Bein) kosti; (Sebirge (od $\mathfrak{B e r g}$ ) horstvo. 3. tvorící adjektiva ze substantiv, aby se naznačila jsouenost věci substantivem vyjádřené: ge= armt (od Wrmt) a s rameny, majici ramena; gebarntifidt (od ఏarnijd) a s krunýřem, mající krunýry. 4. tvoŕíci verba z jiných verb: ges falfen (od fallen) $v_{n}$ líbiti se; gerimen (od rinnert) $\psi_{n}$ srážeti se. 5. tvoíicí part. $p$.: geadjtet (od adjtent) ctil, ctěn; v prov. a poet. nĕkdy vynecháno; fomtntert, worben misto gefonmen, getworben. 6 . F hrs v kuleẻnsk $(\stackrel{t}{)}=$ gemadit.

Abb. 1: STERZ, S. 1011

Ge-[e], auch: -ge-e (Typ.: Nachgelaufe), das; -s: drückt das sich wiederholende [lästige o. ä.] Tun oder Geschehen aus /verbales Basiswort; ausgenommen sind solche Verben, die keine verbalen Formen mit ge- bilden können: Verben mit untrennbarer Vorsilbe (also nicht: Geentwerte, dafür: Entwerterei), Verben mit einer trennbaren Vorsilbe vor einem fremdsprachlichen Verb (also nicht: Nachgepoliere, dafür Nachpoliererei) und Verben, die aus zusammengesetzten Substantiven abgeleitet sind (also nicht: Geschulmeistere, dafür: Schulmeisterei)/ (oft abwertend): Gefeilsche, Gefrage, Geklotze, Gelabere, Geschrei[e], Gesuche, Gesülze, Gezappel[e]; in Verbindung mit Verben mit trennbarer Vorsilbe: das Großgetue, Großgeschreibe, Mitgebringe, Mitgeklatsche, Nachgepfeife, Vorgesage, Zugeknalle; /im Unterschied zu -[er]ei auch in Verbindung mit Verben, deren Subjekt kein belebtes Wesen ist/ Gedonner[e], Geratter[e]. sinnv.: $\uparrow$-[er]ei (Feilscherei). 
ent-〈verbales Präfix 〉: 1. /drückt aus, daß etwas wieder rückgängig gemacht, in den Ausgangszustand zurückgeführt wird/ a) /Ggs. ver-/: entgesellschaften, sich entlieben, (sich) entloben, entkorken, entkrampfen, entmieten, entschlüsseln, entsiegeln, entstofflichen, entzaubern, entzerren. sinnv.: weg-. b) /Ggs. be-/: entkleiden, entladen, entvölkern, entwaffnen, sich entweiben. sinnv.: ab-, aus-. c) /Ggs. das betreffende Grundwort/: entadeln, entdiskriminieren, entflechten, entionisieren, entknoten, entkuppeln, entmotivieren, entsichern, entspannen (ein Gewehr), entsperren (das Sparbuch entsperren), enttarnen, entwarnen. d) / drückt aus, daß das im Basiswort Genannte aus/von etwas entfernt, von etwas befreit wird/: entästen, entblähen, entbörsen (man hatte sie entbörst $=i h r$ die Börse gestohlen), entflimmern, entgiften, entgräten, entgrenzen, enthaupten, enthülsen, entjungfern, entkeimen, entkernen, entknittern, entlausen, entmotten, entölen, entrunzeln, entrüsten ( $=$ Rüstung abbauen), entschärfen, entschwefeln, entspitzen (Pflanzenteile e.), entstempeln (ein entstempeltes Kennzeichenschild), entstören, entwässern. e) /oft in Verbindung mit einem fremdsprachlichen Basiswort, an dessen Stamm meist -isieren angehängt wird, z. B. entpolitisieren (aus: ent-polit[isch]-isieren), entprivilegisieren [aus: entprivileg-isieren]/ machen, daß das Objekt nicht mehr von dem im Basiswort Genannten bestimmt, beherrscht wird, nicht mehr so ist: entbürokratisieren, entdemokratisieren

(immer weniger demokratisch machen), entdramatisieren, enterotisieren, enthierarchisieren, entideologisieren, entindividualisieren, entkonkretisieren, entkriminalisieren (vom Kriminellen befreien), entmystifizieren, entnazifizieren, entnuklearisieren, entproblematisieren, entsakralisieren, entstalinisieren, enttabuisieren, entterminologisieren. 2. 〈verstärkend > machen, daß es so wird, wie es das Basiswort angibt: entblößen, entleeren. 3. /drückt aus, daß ein Vorgang, eine Handlung beginnt, einsetzt/: entbrennen, entfachen, sich entspinnen, entflammen. 4. a) /drückt aus, daß sich jmd./etwas von etwas entfernt/: enteilen, entfliehen, entgleiten, enthüpfen, entlaufen, entschreiten, entschweben, entspringen, entweichen. b) /kennzeichnet, daß etwas aus etwas heraus und zu einer anderen Stelle gelangt/: entleihen. c) /drückt aus, daß etwas aus etwas herausgelangt/: entlocken, entnehmen, entreiBen, entsteigen. $\square($ od $\mid$ čarovat, vy|prázdnit, $u \mid$ prchnout ...)

Abb. 6: DUDEN, S. 219

\section{Anhang II: Substantive auf Ge-...(-e) (Neutra): Bestandaufnahme - einschließlich lexikalisierter und idiomatischer Bildungen sowie unproduktiver Typen (Quelle: DUW, CCDB, DeReWo)}

Im Fettdruck stehen Lexeme, die synchron (auch) als (relativ) durchsichtige Wortbildungen nach dem produktiven Wortbildungsmodell (Nomina actionis) gedeutet werden können. Die Verwendung des jeweiligen Wortbildungsprodukts in einer bestimmten Lesart ist stets stark 
diskursbezogen, weswegen es auch mehreren Wortbildungsmodellen zugeordnet werden kann (vgl. z. B. den fließenden Übergang zwischen Nomina actionis und Nomina acti). Das Zeichen * (vor einem Lexem) bezeichnet die in der CCDB vorgefundenen, doch im DUW nicht inventarisierten Wortbildungsprodukte, die im Falle des korpusgesteuerten bzw. korpusbasierten GAWDT als Lemmakandidaten aufzufassen sind. Das Zeichen ! (vor einem Lexem) steht bei den im derewo-v-30000g-2007-12-31-0.1 (siehe unter www.idsmannheim.de/k1/projekte/methoden/derewo.html) verzeichneten Lexemen, die somit zu den 30000 häufigsten Grundformen im DeReKo zählen, und deshalb besondere Aufmerksamkeit verdienen. Bei Homonymen Gehalt, Gericht, Geschick, Geschirr und Geschoß/Geschoss bezieht sich die Frequenzangabe summativ auf die Vorkommenshäufigkeit beider Konstituenten des jeweiligen homonymischen Lexempaares.

Geächze Geäder Geäfter Gealber Gealbere Geäse Geäst Gebabbel !Gebäck Gebalge Gebälk Geballer Geballere Gebände Gebäu !Gebäude !Gebein Gebell Gebelle Gebende !Gebet Gebettel *Gebild !Gebilde Gebimmel Gebinde !Gebirge !Gebiss *Gebiß Geblaff Geblaffe Geblase Gebläse Geblödel Geblök Geblöke Geblüt*Gebolze !Gebot Gebrabbel Gebräch Gebräche Gebräu Gebraus Gebrause Gebrech Gebreche Gebreit Gebreite Gebröckel Gebrodel Gebrüll Gebrülle Gebrumm Gebrumme Gebrummel Gebumse !Gebüsch Gedärm Gedärme Gedeck !Gedicht Gedinge Gedonner Gedöns !Gedränge Gedrängel Gedröhn Gedröhne Gedudel Gefach Gefahre !Gefährt !Gefälle Gefasel !Gefäß Gefauch Gefauche !Gefecht Gefege Gefeilsch Gefeilsche Gefiedel Gefieder !Gefilde Geflacker Geflackere Geflatter Geflattere !Geflecht Geflehe Geflenne Geflimmer Geflimmere Geflitz Geflitze Gefluche !Geflügel Geflunker Geflunkere Geflüster !Gefolge Gefrage Gefrotzel Gefuchtel Gefuchtele !Gefüge !Gefühl Gefummel Gefummele Gefummle Gefunkel Gegackel Gegackele Gegacker Gegeifer Gegirr Gegirre Geglitzer Geglitzere Geglucks Gegluckse Gegrinse Gegröl Gegröle Gegrunz Gegrunze !Gehabe !Gehalt *Gehämmer *Gehämmere *Gehampel Gehänge Gehänsel Gehänsele !Gehäuse Geheck !Gehege !Geheiß Gehetz Gehetze Geheul *Geheule !Gehirn !Gehöft Geholper Geholpere !Gehölz Geholze Gehoppel Gehoppele Gehops Gehopse !Gehör Gehörn Gehudel Gehudele Gehupe Gehüpfe Gehuste Gejammer Gejammere Gejauchze Gejaul Gejaule Gejodel Gejohl Gejohle Gekeif Gekeife Gekeuche Gekicher Gekläff Gekläffe Geklapper Geklatsch Geklatsche Geklimper Geklimpere Geklingel Geklingele Geklingle Geklirr Geklirre Geklopf Geklopfe Geklüft Geklüfte Geknall Geknalle Geknatter Geknirsch Geknirsche Geknister Geknutsche Gekrabbel Gekrabbele Gekrächz Gekrächze Gekrakel Gekrakele Gekrakle Gekrätz Gekratze Gekräusel Gekreisch Gekreische Gekritzel Gekritzele Gekröse Gekungel Geküsse Gelabber Gelaber Gelabere Gelache !Gelächter Gelage Geläger *Gelalle !Gelände !Geländer Gelärm Gelärme Gelass *Gelaß Geläster Geläuf Gelaufe Geläut Geläute Gelege Geleier !Geleise !Geleit Geleite !Gelenk Gelispel Gelübde Gelump Gelumpe Gelüst Gelüste Gemach Gemächt Gemächte !Gemälde !Gemäuer Gemaule Gemauschel Gemecker Gemeckere Gemenge Gemengsel !Gemetzel !Gemisch Gemotze Gemuffel Gemunkel Gemurmel *Gemurre !Gemüse !Gemüt !Genick *Genöle Genörgel !Gepäck Gepfeife *Gepiepe *Gepiepse !Geplänkel Geplapper *Geplärr *Geplärre Geplätscher Geplauder Gepolter Gepräge Gepränge *Geprassel *Gequake *Gequäke *Gequassel Gequatsche *Gequengel *Gequieke *Gequietsche *Geraffel !Gerangel *Geraschel *Gerassel !Gerät*Geraune *Geraunze 
!Geräusch !Gerede Gereime *Gerenne !Gericht Geriesel *Gerinne Gerinnsel Gerippe *Geröchel !Geröll Gerölle !Gerücht Gerufe Gerumpel Gerümpel !Gerüst *Gerüttel *Gesabber !Gesäß *Gesäuge *Gesäusel *Geschacher !Geschäft Geschäker Gescharre Geschaukel!Geschenk Geschepper !Geschick Geschiebe Geschieße Geschimpfe !Geschirr Geschiss Geschlabber !Geschlecht *Geschlinge Geschmeide Geschmeiß *Geschmetter Geschmier Geschmiere *Geschmuse *Geschnatter !Geschöpf Geschoß !Geschoss !Geschrei *Geschreibe *Geschubse !Geschütz Geschwader Geschwafel !Geschwätz Geschwatz Geschwatze Geschwätze !Geschwister Geschwür !Gesetz !Gesicht Gesims Gesinde Gesindel Gesinge Gesocks Gesöff !Gespann !Gespenst Gespinst Gespött !Gespräch !Gespür Gestade Gestammel *Gestampfe Gestänge Gestänker Gesteck !Gestein !Gestell Gestirn *Gestöber Gestöhn Gestöhne *Gestolper Gestotter *Gestrampel Gesträuch Gestrick !Gestrüpp Gestühl *Gestürm !Gestüt !Gesuch *Gesülze Gesumm Gesumme *Getändel Getier *Getobe Getön Getöne Getose !Getöse *Geträller Getrampel !Getränk *Getrappel Getratsch Getratsche !Getreide !Getriebe *Getrippel Getrommel Getu Getue !Getümmel Getuschel Geviert Gewackel Gewackele Gewackle !Gewächs Gewaff Gewaffen !Gewand Gewände Gewann Gewanne Gewäsch !Gewässer !Gewebe !Gewehr Geweih Geweine !Gewerbe Gewerk Gewese Gewieher !Gewicht Gewimmel Gewimmer Gewinde Gewinsel Gewirbel Gewirk Gewirke !Gewirr Gewirre Gewisper !Gewitter Gewitzel Gewoge !Gewölbe Gewölk Gewölle Gewühl Gewürge Gewürm !Gewürz Gewusel Gezänk Gezanke Gezappel !Gezerre Gezeter *Geziefer Geziere Gezirp Gezirpe Gezisch Gezische Gezischel *Gezücht Gezüngel Gezweig Gezweige Gezwitscher

\section{Anhang III: Nomina actionis auf Ge-...(-e) und deren Äquivalente - Ergebnisse einer InterCorp-Recherche}

Die Übersicht umfasst die Wortbildungen auf $G e-\ldots(-e)$, die (auch) als Nomina actionis gedeutet werden können, und ihre tschechischen Äquivalente, die mithilfe des InterCorp eruiert worden sind. Die lokalen syntagmatischen Kontexte konnten aus Platzgründen nur in sehr eingeschränktem Ausmaß aufgenommen werden. "Misz[ellen]" ist ein Sammelbegriff für (a) solche Fälle, in denen dem deutschen Substantiv auf der tschechischen Seite kein direktes Äquivalent gegenübersteht: Eines Tages hatte Wagner plötzlich gebrüllt und drei Tage lang mit Brüllen [Subst.] nicht mehr aufgehört. Wagner se jednoho dne zničehonic rozkřičel a po celé tři dny křičet [Verb] neprestal und für (b) verschiedene Auslassungen und Zweifelsfälle (Stand: 2011).

Gebabbel dýchánky 1X Geballer střelba 1X Gebell štěkot 14X štěkání 2X Misz. 1X Gebet modlitba $124 \mathrm{X}$ modlitby $12 \mathrm{X}$ modlitbička $3 \mathrm{X}$ zaklínadlo $3 \mathrm{X}$ prosby $2 \mathrm{X}$ hosana $1 \mathrm{X}$ mše $1 \mathrm{X}$ výzva $1 X$ volání $1 X$ zaklínání $1 X$ zaříkávadlo $1 X$ Misz. 45X Gebettel žadonění $1 X$ Gebimmel cinkání 1X Misz. 3X Geblöke Misz. 1X Gebrabbel blábolení 2X kvílení 1X Gebrüll řev $44 X$ řvaní $11 X$ zařvání $3 X$ bučení $3 X$ (krav - G. der Kühe) povyk $2 X$ pokřik $2 X$ hulákání $1 \mathrm{X}$ vřeštění $1 \mathrm{X}$ křik $1 \mathrm{X}$ výkřiky $1 \mathrm{X}$ ryk $1 \mathrm{X}$ mnohonásobné ryčné výkřiky $1 \mathrm{X}$ (G. Und Geschrei) Misz. 7X Gebrumm brumlání 1X bručení 1X mručení 1X bzukot 1X hukot $1 \mathrm{X}$ Gedonner děsivost $1 \mathrm{X}$ Gedränge tlačenice $30 \mathrm{X}$ zástup $2 \mathrm{X}$ mačkanice $2 \mathrm{X}$ nával $1 \mathrm{X}$ skupina 1X hemžení 1X chumel 1X dav 1X Misz. 8X Gedröhn hluk 1X hučení 1X dunění $1 \mathrm{X}$ Gefasel blouznění $3 \mathrm{X}$ blábol $1 \mathrm{X}$ blábolení $1 \mathrm{X}$ bláboly $1 \mathrm{X}$ žvásty $1 \mathrm{X}$ žvást $1 \mathrm{X}$ pošetilost 1X třeštění 1X Gefiedel fidlání 1X Geflatter Misz. 4X Geflimmer pohyb 1X (der Farben: 
barev) blyskot $1 X$ Geflüster šepot 14X šeptání 5X šuškání 2X Misz. 3X Gefrage otázka 1X Gefunkel jiskření 1X třpyt 1X Misz. 1X Gegacker kokodání 1X slepičí nářky 1X pokřik 1X křik 1X Gegröle zpěvy 1X Gehämmer bušení 4X Gehetze spěch 1X štvaní 1X Geheul vytí $6 \mathrm{X}$ řev $4 \mathrm{X}$ skučení $2 \mathrm{X}$ zaskučení $1 \mathrm{X}$ kvílení $1 \mathrm{X}$ vřesk $1 \mathrm{X}$ nářek $1 \mathrm{X}$ jásot $1 \mathrm{X}$ jekot $1 \mathrm{X}$ ječení 1X ječivé výkřiky 1X pláč 1X sten 1X štkaní 1X Misz. 3X Geholper Misz. 1X Gehops tanec 1X Gehopse křepčení 2X Gehüpfe poskakování 1X Gehuste kuckání 1X kašel 1X Gejammer nářky $3 X$ nářek $2 X$ naříkání $1 X$ skuhrání 1X Misz. 1X Gejammere Misz. 1X Gejaule nářek 1X Misz. 1X Gejohle řev $1 \mathrm{X}$ jódlování $1 \mathrm{X}$ výskání $1 \mathrm{X}$ vř́skot $1 \mathrm{X}$ Gekeif nadávky 1X Gekeife křik 1X jekot 1X Gekeuche hekání 2X Gekicher chichotání 2X smích 2X hejkání 1X Misz. 1X Gekläff štěkot 4X Misz. 4X Gekläffe štěkání 1X Geklapper vrzání 1X Misz. 3X Geklingel cinkání 1X klinkání 1X zvonění 1X Geklirr zvonění 1X řinkot 1X řinčení 1X Geknall švihání 1X Geknalle střelba 2X Geknatter třesknutí 2X třeskot 1X salvy $1 X$ (rýmů - der Reime) znamení 1X (sirény - G. der Flak) střelba 1X klapání 1X práskání 1X Gekrach třesk 1X Gekrächze řev 1X škručení 1X Gekrakel čmáranice 1X Gekräusel řasení $1 X$ Gekreisch jek $3 X$ jekot $2 X$ křik $2 X$ skřeky $1 X$ výkřiky $1 X$ ječení $1 X$ skřek $1 X$ vř́skot $1 X$ kravál 1X skučení 1X Gekritzel klikyhák 1X čárky 1X Geküsse Misz. 1X Gelaber žvásty $1 X$ Gelache legrace $2 X$ Gelärme nezbednosti $1 X$ Geläute zvonění $2 X$ vyzvánění $1 X$ Misz. 2X Gemaule Misz. 1X Gemetzel jatka $8 X$ krveprolití $6 X$ masakr 4X řež 2X řeže 1X potoky krve $1 X$ vraždění $1 X$ řezničina $1 X$ Misz. 3X Gemurmel mumlání $9 X$ šepot $8 X$ mručení $5 X$ šeptání $1 X$ brumlání $1 X$ vřava $1 X$ šum $1 X$ šelest $1 X$ Misz. $5 X$ Gepfeife pískot $3 X$ hvízdání $1 \mathrm{X}$ Geplänkel přestřelka $3 \mathrm{X}$ potyčka $1 \mathrm{X}$ dovádění $1 \mathrm{X}$ zápolení $1 \mathrm{X}$ hašteření $1 \mathrm{X}$ záležitost $1 \mathrm{X}$ Geplärr řev 1X Geplärre řev $1 X$ brek $1 X$ Geplätscher šplouchání $1 X$ hrčení $1 X$ bublání $1 X$ Misz. 2X Geplauder tlachání 2X povídání 1X rozhovor 1X Misz. 1X Gepolter bouření 1X Geprassel praskání $2 \mathrm{X}$ palba $1 \mathrm{X}$ praskot $1 \mathrm{X}$ rachot $1 \mathrm{X}$ šumění $1 \mathrm{X}$ Gequake kvákání $1 \mathrm{X}$ Gequassel řeči $2 X$ řeč $1 X$ Misz. 1X Gequatsche blábol $1 X$ bláboly $1 X$ žvanění $1 \mathrm{X}$ řeči $1 \mathrm{X}$ kecání 1X Misz. 1X Gequieke kňučení 1X Gerangel tahanice 2X šarvátky 1X broušení ostruh $1 \mathrm{X}$ volná tribuna $1 \mathrm{X}$ tartas $1 \mathrm{X}$ vřava $1 \mathrm{X}$ křŕžkování $1 \mathrm{X}$ proplétání $1 \mathrm{X}$ Gerassel Misz. 1X Geraune brumlání 1X Misz. 1X Gerede řeči 39X řeč 4X žvanění 4X řečnění 3X slova $3 \mathrm{X}$ kecy $3 \mathrm{X}$ projevy $2 \mathrm{X}$ debaty $2 \mathrm{X}$ rétorika $1 \mathrm{X}$ skandál $1 \mathrm{X}$ povídačky $1 \mathrm{X}$ pomluvy $1 \mathrm{X}$ pověst $1 \mathrm{X}$ teorie $1 \mathrm{X}$ (Lass das G. - Nech stranou teorii.) řečičky $1 \mathrm{X}$ diskuse $1 \mathrm{X}$ hlóposti $1 \mathrm{X}$ žvásty $1 \mathrm{X}$ tlachy $1 \mathrm{X}$ pověry $1 \mathrm{X}$ plky $1 \mathrm{X}$ hovor $1 \mathrm{X}$ klepy $1 \mathrm{X}$ plané povídání $1 \mathrm{X}$ plané tlachání $1 \mathrm{X}$ prázdná slova $1 \mathrm{X}$ jalové žvásty $1 \mathrm{X}$ hlas lidu $1 \mathrm{X}$ Misz. 20X Gerenne shon $1 \mathrm{X}$ dusot rychlých kroků 1X dupot utíkajících nohou 1X Misz. 3X Geriesel Misz. 1X Gerüttel třas 1X drnčení 1X Gesabber slintání 2X Gesäusel útlocitnosti 1X Geschacher čachry 1X Gescharre šoupání 1X Geschaukel houpačka 1X Misz. 1X Geschepper rozruch 1X rachot 1X Geschiebe Misz. 1X Geschieße střelba 2X přestřelka 1X Geschimpfe nadávky 1X Misz. 3X Geschnatter brebentění $2 X$ kvákání $1 X$ pokřik $1 X$ Geschrei křik $123 X$ pokřik $21 \mathrm{X}$ řev $20 \mathrm{X}$ výkřiky $8 \mathrm{X}$ povyk $5 \mathrm{X}$ hluk $4 \mathrm{X}$ výkřik $4 \mathrm{X}$ volání $4 \mathrm{X}$ křiky $4 \mathrm{X}$ jekot $3 \mathrm{X}$ řvaní $3 \mathrm{X}$ nářek $2 \mathrm{X}$ pokřikování $2 X$ vřava $1 X$ zpráva $1 X$ vyvolávání $1 X$ hřmění $1 X$ volání $1 X$ hučení $1 X$ halekání $1 \mathrm{X}$ jek $1 \mathrm{X}$ hulákání $1 \mathrm{X}$ hněv $1 \mathrm{X}$ řehot $1 \mathrm{X}$ mela $1 \mathrm{X}$ vytí $1 \mathrm{X}$ překřikování $1 \mathrm{X}$ řeči skřek (das schrille G.) $1 X$ zařvaní $1 X$ křičení $1 X$ vřesk $1 X$ pláč $1 X$ hlas $1 X$ skřeky $1 X$ Misz. 29X Geschubse hrátky $1 X$ Geschwafel bláboly $1 X$ Geschwätz řeči $5 X$ tlachání $4 X$ žvásty $4 X$ žvanění $2 X$ tlachy $3 X$ kecy $2 X$ žvást $2 X$ plané řeči $1 X$ bláboly $1 X$ šumy $1 X$ plky $1 X$ pomluvy $1 \mathrm{X}$ báchorky $1 \mathrm{X}$ klepy $1 \mathrm{X}$ žvatlání $1 \mathrm{X}$ povídání $1 \mathrm{X}$ chvástání $1 \mathrm{X}$ blábol $1 \mathrm{X}$ povídačka $1 \mathrm{X}$ nezaručené pověsti a podobné kecy $1 X$ tlach $1 X$ Misz. $2 X$ Gespött posměch $7 X$ výsměch $2 X$ 
posměváčkovství $1 \mathrm{X}$ úsměšek $1 \mathrm{X}$ předmět pobavení $1 \mathrm{X}$ terč posměchu $1 \mathrm{X}$ objekt posměchu 1X Misz. 7X Gestammel koktání 1X vzlyky 1X žbrblání 1X Gestampfe dusot 1X dupot 1X Gestöber vánice $2 X$ poprašek 1X snopy 1X Misz. 2X Gestöhn sténání 1X Gestöhne Misz. 1X Gesülze Misz. 1X Gesumm bzukot 2X cvrlikání 1X Gesumme Misz. 1X Getändel laškování 1X Getobe povyk 1X Getön Misz. 1X Getöse rámus $8 X$ rachot $8 X$ hluk $5 X$ hřmot $4 \mathrm{X}$ povyk $4 \mathrm{X}$ řev $3 \mathrm{X}$ řvaní $3 \mathrm{X}$ hukot $2 \mathrm{X}$ fanfáry $2 \mathrm{X}$ chvástání $1 \mathrm{X}$ vychvalování $1 \mathrm{X}$ šílenství $1 \mathrm{X}$ hlomoz $1 \mathrm{X}$ změt' $1 \mathrm{X}$ cinkání $1 \mathrm{X}$ randál $1 \mathrm{X}$ šumění $1 \mathrm{X}$ potlesk $1 \mathrm{X}$ ryk $1 \mathrm{X}$ třeskot $1 \mathrm{X}$ dunění $1 \mathrm{X}$ vřava $1 \mathrm{X}$ lomoz $1 \mathrm{X}$ zahřmění $1 \mathrm{X}$ hřmění $1 \mathrm{X}$ hukot a hřmot $1 \mathrm{X}$ vyzvánění $1 \mathrm{X}$ (der Kirchenglocken) Misz. 8X Geträller zpěv 1X Getrampel dupot 3X dupání 2X dusot 1X Getrappel dusot $3 X$ dupot $2 X$ Misz. 1X Getriebe soukolí $7 X$ převodovka $3 X$ ruch $2 X$ kola $1 X$ převod $1 X$ hlučný ruch $1 X$ veškerý ruch $1 X$ ruch $1 X$ (Lärm u. G.) pohon $1 X$ shon $1 X$ mechanismus 1X Getrippel cupitání 1X Getrommel rámus 1X (G. u. Getröte) bubínek 1X Getümmel shon $2 X$ vřava $2 X$ šarvátka $1 X$ dav $1 X$ řež $1 X$ tlačenice $1 X$ hluk $1 X$ zmatek $1 X$ rvačka $1 X$ (Geschrei u. G.) Misz. 1X Getuschel šuškání $2 X$ řeči $1 X$ posměch $1 X$ žerty $1 X$ šeptání 1X šepot 1X Misz. 1X Gewieher smích 1X řehot 1X Gewimmer kvílení 1X zakvílení 1X Gewinsel vytí 3X kňučení 1X vrnění 1X Misz. 1X Gewirbel vír 1X Gewisper šepot 1X Gewoge vlnění $1 \mathrm{X}$ Gewühl chumel $4 \mathrm{X}$ dav $3 \mathrm{X}$ tlačenice $2 \mathrm{X}$ lidé $1 \mathrm{X}$ shluk $1 \mathrm{X}$ hemžení 1X zástup 1X sevření 1X Misz. 1X Gewürge handrkování 1X Gewusel hemžení 1X Gezänk hašteření $6 X$ hádky $3 X$ spory $2 X$ pletichy $1 X$ hádka $1 X$ Misz. 1X Gezappel hupkání 1X Misz. 1X Gezerre tahanice 1X Gezeter řeči 2X nářky $1 X$ křičení $1 X$ výkřiky $1 X$ křik $1 X$ pronikavý jek 1X Misz. 1X Gezirp šeptání 1X Gezisch syčení 1X Misz. 1X Gezischel šum 1X Misz. 1X Gezwitscher štěbetání 2X pípot 1X švíření 1X pípání $1 X$ hlasy $1 X$

\section{Anhang IV: Nomina actionis auf Ge-...(-e): Ausgewählte GAWDT-Datenbankoutputs (vorläufige Versionen, Stand: April 2013) entsprechenden SIEB-Wörterbucheinträgen gegenübergestellt}

Das Siebenscheinsche Wörterbuch von 2006 (weiter nur SIEB) ist das umfangreichste (relativ) aktuelle deutsch-tschechische Übersetzungswörterbuch. Deshalb stellt es aus der Sicht der Bearbeiter von GAWDT-Inputs eine bedeutende Bezugsgröße dar, an der die Wörterbucheinträge des entstehenden Wörterbuchs quantitativ wie qualitativ gemessen werden. Praktischer Nutzen der am GAWDT getesteten korpusanalytischen Methoden wird durch die Gegenüberstellung von SIEB- und GAWDT-Wörterbucheinträgen bereits bei einer oberflächlichen Betrachtung klar ersichtlich. Legende: subst[antivum] - Substantiv, též hanl[ivě] - auch abwertend, neform[álně] - informell, přen[eseně] - übetragen, synonyma Synonyme, slovotvorba - Wortbildung, mys1[ivecký] jaz[yk] - Jägersprache.

s Gebẹll (-[e]s, 0) štěkot

Abb. 7: SIEB, S. 568

das Gebell, Gebelle, Ge|b\#ell, Ge|b\#el|le
subst.
-(e)s, $0 ;-$ s, 0
též hanl.
A štěkot


štěkání

hafání neform.

übermässiges, fortwährendes Gebell und Geheul intenzivní a neustálý štěkot s vytím

Gegen drei hat mich ein lautes/wütendes/wildes/freudiges Gebell meines Hundes geweckt. Kolem třetí mě probudil hlasitý/zuřivý/divoký/radostný štěkot mého psa.

B pren.

štěkot

štěkání

das Gebell der Maschinengewehre štěkot kulometů

SYNONYMA

das Bellen, die Bellerei

SLOVOTVORBA

A, B $\uparrow$ Ge-...(-e) A

$\uparrow$ bellen, das Bellen, die Bellerei

$\uparrow$ das Hundegebell

Abb. 8: (C) GAWDT (Datenbankoutput Gebell)

s Geläute (-s, 0) zvonění, vyzvánění; plötzlich er-

klang ein mächtiges $\mathrm{G}$. der Glocken náhle se

mocně rozezvučely zvony; s letzte $\mathrm{G}$. zvonéni pri

pohřbu

Abb. 9: SIEB, S. 581

Geläute, Ge|1_ä_u|te

subst.

$-\mathrm{s}, 0$

A

zVonění

vyzvánění

hlahol

Nach halb sieben war von der Hauptstraße das Geläute der Glocken zu hören. Po půl sedmé bylo od/z hlavní třídy slyšet vyzvánění zvonů.

B

(kostelní) zvony

soustava, skupina zvonů

Ins neue Geläute wurden zwei Glocken aus dem 17. Jh. integriert. Do nové soustavy zvonů byly integrovány dva zvony ze 17. stol.

C mysl. jaz.

štěkot loveckých psů

štěkání psů na lovu

štěkot (psí) smečky

Jede Hundemeute hat ihr eigenes Geläute. Každá smečka loveckých psů se vyznačuje svým vlastním typickým štěkotem. 


\section{SYNONYMA}

A anhaltendes Läuten, $\uparrow$ Geläut $B, C \uparrow$ Geläut

\section{SLOVOTVORBA}

$\mathrm{A} \uparrow \mathrm{Ge}-\ldots(-\mathrm{e}) \mathrm{A}$

个läuten

Abb. 10: () GAWDT (Datenbankoutput Geläute)

s Geplạuder (-s, 0) povídání, tlachání

Abb. 11: SIEB, S. 587

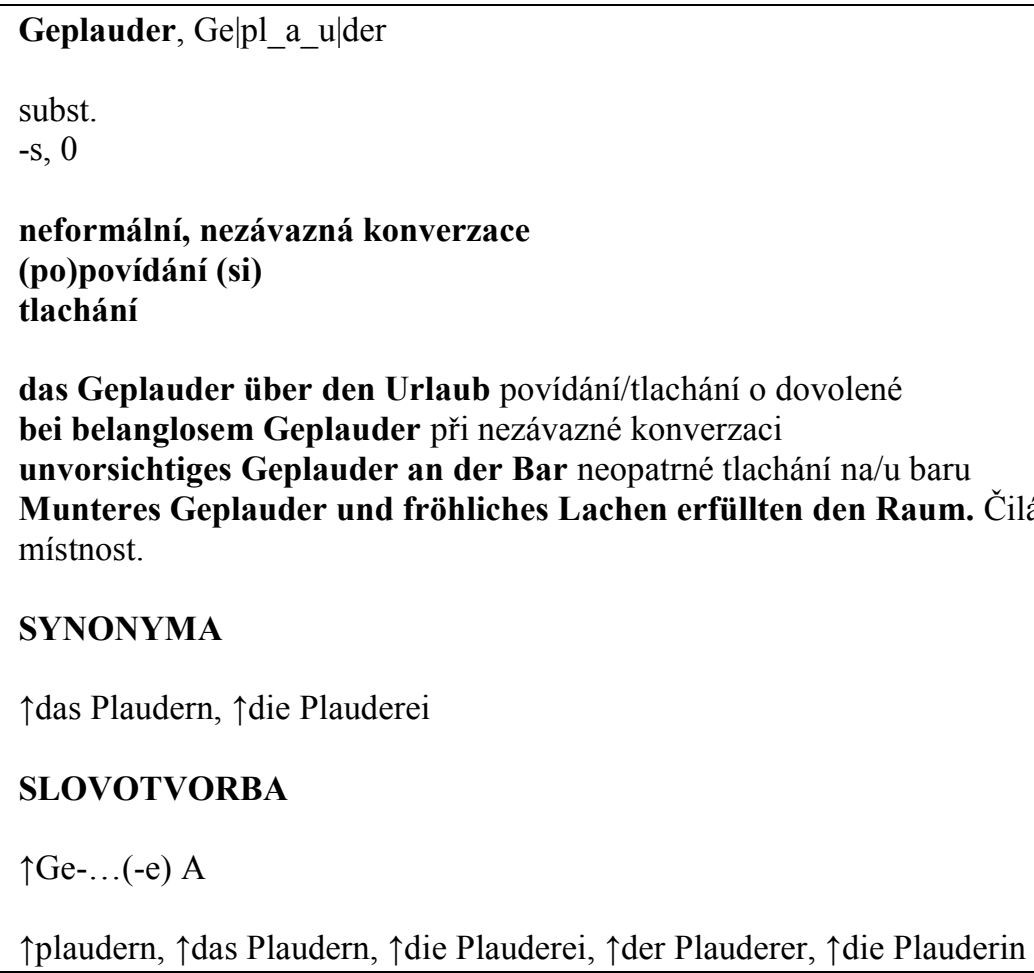

Abb. 12: @ GAWDT (Datenbankoutput Geplauder) 